\title{
Las razones geológicas de la minería del cobre
}

\author{
L. de la Torre y J. A. Espí \\ Universidad Politécnica de Madrid. Escuela Técnica Superior de Ingenieros de Minas y Energía. Rios Rosas 21. 28003 Madrid. \\ Itp@coimce.com \\ joseantonio.espi@upm.es
}

\section{RESUMEN}

\begin{abstract}
A pesar de encontrarse el debate abierto desde mediados del siglo pasado, existe un todavía débil conocimiento sobre la existencia real de los minerales tecnológica y económicamente accesibles. Para salir de esta situación, resulta necesario comenzar mejorando la información geológica en muchos aspectos, desde la precisión de la base cartográfica en zonas prometedoras, a la elaboración de modelos más precisos de aparición de mineralizaciones $y$, sobre todo, de sus controles geológicos. El paulatino descenso de las leyes explotadas, así como una producción en continuo crecimiento, acercan al debate la lejana amenaza del agotamiento, apartada del contexto real o geológico. Respecto al futuro, existen dos vertientes de opinión muy diferenciadas: analistas que ven los precios de las materias primas minerales estacionarios a largo plazo, concluyendo que la escasez de los metales, en general, no será de momento ningún problema. Otros, sin embargo, concluyen que la relación de precios con el tiempo posee una forma de $U$ y, por lo tanto, la escasez estaría en aumento a largo plazo. Sin embargo, durante el pasado siglo y en periodos de explosión del consumo, no ha existido nunca problema alguno de escasez, en todo caso, una pérdida temporal del balance oferta-demanda.
\end{abstract}

Palabras clave: cobre, economía mineral, geología económica, recursos minerales.

\section{Making sense of the geology in the copper mining economy}

\begin{abstract}
Despite the ongoing debate since the middle of the last century, there is still a lack of knowledge about the real existence of the technologically and economically accessible minerals. To overcome this situation, it is necessary to start improving geological information in many aspects, from the precision of the cartographic knowledge in promising areas, to the development of more precise patterns of mineralization and, above all, to their geological controls. The gradual decrease in the metal grades exploited, as well as a production undergoing continuous growth, brings the distant threat of exhaustion to the debate, which has become separated from the real or geological context. Regarding the future, there are two very different points of view: analysts who see the prices of mineral raw materials as being stationary in the long term, concluding that the shortage of metals, in general, will not be a problem at the moment. Others, however, conclude that the price relationship over time has a U-shape and, therefore, scarcity will increase in the long run. However, during the last century and in periods of a surge in consumption, there has never been any problem of scarcity, merely a temporary loss of supply-demand balance.
\end{abstract}

Keywords: copper, geological economy, mineral economy, mineral resources.

\section{ABRIDGED ENGLISH VERSION}

\section{Introduction}

Despite the open debate since the middle of the last century, there is still a lack of knowledge about the real existence of technologically and economically accessible minerals. To overcome this situation, it would be necessary to start improving geological information in many aspects, from the precision of the cartographic base to promising areas, to the development of more precise patterns of mineralization and, above all, to their geological controls. In this context, the activity of the North American Geological Survey (USGS) (biblio- 
graphy ...) has advanced the methodology of prediction of undiscovered mineral resources, always within a broad conception of the metalogenetic models and the conditions, both of their appearance and discovery, and of the practical circumstances of their real and economic use.

\section{Results and discussion}

Copper, as far as value is concerned, is the third metal in world production after iron and aluminum. The importance of this basic metal (Fig. 1) is shown by the trading on the international stock exchanges (LME, NYME, Shanghai, etc.) along with the main raw materials, participating in spot prices, as well as in the futures market.

It has been observed that in the last century the recognized volume of copper grew by about 25 times (Schodde, 2010). The reasons for this tremendous growth in a recognized resource can be found, apart from a successful exploration and price, in the natural mechanism of the conversion of resources-reserves. The reduction of the limit average of mine grades over time, or the relationship of the grade with tonnage, facilitates the resource-reserve validation mechanism. Thanks to the technology applied we are able to better understand the feasibility of these new deposits with much lower grades, cost reduction and greater accessibility.

The basic tools are the production cost curves, expressed by mineral substances or even better, by specific metals. In any case, the application of curves to the world of copper is very complete and easily accessible from different sources. As far as global copper deposits are concerned, including all their typologies and sizes, high grades or near-surface deposits are very difficult to obtain today, especially deposits with a low open-pit mining rate that would allow low operating costs. Deeper deposits require underground mining methods which, in general, mean much higher operating costs.

Achieving a project within the 50th percentile of the cost curve is increasingly complicated, since the known reservoirs with higher grades are generally deeper and the undeveloped ones have very low grades (Fig. 10).

There will be very few projects that are able to overcome the obstacles of at least $15 \%$ IRR and, for longterm security, that are within the 50th percentile of the cost curve, with the security that this entails in the case of falling prices. In any case, there will be a considerable delay before projects that meet the above conditions are ready for production. This delay is inevitable for a time, during which, the metal supply will be reduced and demand will gradually or even drastically increase. This will lead to the next increase in the metal price. The only thing that is uncertain in this scenario is when it will happen, as history has recorded on several occasions.

This mechanism tends to eliminate projects with higher extreme costs as a result of quoted price reductions and includes the elimination of low-cost projects by accelerated depletion or due to their small size. The achievement of a reduction in costs in recent years to continue to remain within the appropriate percentile has usually originated thanks to the new technologies that appeared between 1905-2007, achieving a cost reduction of up to $70 \%$ in that period (see Fig. 8). Another significant parameter in reducing mine costs is the economies of scale, which in the same period reached a $30 \%$ improvement.

The current technology helps metallogenic types or models that best adapt to the new demands of the mining industry. This is the case of mine gigantism, in an effort to exceed in some moments the low prices and take advantage of the facilities that typologies present such as copper porphyries. Today, efforts to find new typologies of copper deposits are evident: although predominantly subterranean, skarns, VMS and, above all, sediment hosted (SH) and IOGC appear to re-emerge in response to the explained causes.

There are other factors: access to new territories, environmental and social pressures and the relative decline in underground operating costs. It does not appear that the production ore grades were totally dependent on market prices. The reason is as follows: it is rather difficult to change the exploitation patterns and an extra capital is necessary which, in the short term, is not is easy to achieve.

The formulation of metalogenetic models arises from the need for an understanding of the phenomenon of its creation in order to facilitate new discoveries. One aspect of the expression of the different types of reservoirs is their association with a particular series of the rocks that are in relationship with them (Stanton, 1972). This association broadly reflects both the geological environment and the processes that have contributed to the formation of the mineral deposit. The identified associations have been widely used in mining exploration.

Geologists generally prefer genetic classification systems that also incorporate elements of their composition, form, and mineral associations. The meaning of the model comes to reflect, not only the genesis of the deposit, but also assumes its economic, technical and productive sense. As an example, for a copper porphyry, when accepting the term, it also assumes its generic conditions of very low open-pit ratio, a more 
L. de la Torre y J. A. Espí, 2019. Las razones geológicas de la minería del cobre. Boletín Geológico y Minero, 130 (1): $133-159$

or less spheroid form, low grades, knowledge or metallurgical domain of its concentration, the grade of its concentrates and several other technical aspects.

\section{Conclusions}

The future evolution of mineral supplies is becoming increasingly complex and evolutionary models are scarcer and of very limited reliability.

At the expert level, there is a huge convergence towards regarding the need for sufficient global geological knowledge to proceed with the development of supply and demand models for all metals, and even more so in the case of copper.

The view is that those mineral resources which are in a location that is now accessible have such abundance that, despite temporary situations of scarcity, do not reach a critical rating. This situation does exist in some cases due to other elements that enter into the production system but is in no way due to the depletion of the mineral stock.

\section{Introducción}

A pesar de lo ampliamente tratado sobre las estimaciones en la producción del cobre, la variabilidad de su nivel de precios y las crecientes e injustificadas amenazas de agotamiento, resulta cuanto menos llamativo lo poco que se ha escrito sobre la base geológica y genética de la producción mundial de cobre. Seguramente, un conocimiento más exhaustivo de las diferentes tipologías de yacimientos y su posible evolución, brindarían una mejor oportunidad para un análisis más cierto de todas estas incertidumbres.

Aunque será el mercado quien marque el límite real del recurso, éste vendrá condicionado por las tipologías en explotación, las leyes extraídas, los costes de operación y, sobre todo, por el éxito de la inversión en tecnología y exploración.

El economista y profesor J.Tilton (2001), figura de referencia en el mundo de la teoría económica de los recursos no renovables, resume la dependencia actual de la economía mineral con las características de su origen natural de la siguiente manera:

[...] "Si la sociedad actual fuera sensible acerca de los temas de la escasez mineral, invirtiendo recursos para aumentar la información geológica a fin de determinar mejor la forma de la curva de suministros acumulados, ello podría proporcionar muchos datos de las circunstancias de una posible reducción de los recursos minerales a largo plazo" (Tilton, 2001).

A pesar de encontrarse el debate abierto desde mediados del siglo pasado, existe un todavía débil conocimiento sobre la existencia real de los minerales tecnológica y económicamente accesibles. Para salir de esta situación, resultaría necesario comenzar mejorando la información geológica en muchos aspectos, desde la precisión de la base cartográfica en zonas prometedoras, a la elaboración de modelos más precisos de aparición de mineralizaciones $y$, sobre todo, de sus controles geológicos. En este orden de cosas, destaca la actividad del Servicio Geológico Norteamericano (USGS) que avanza en la metodología de predicción de recursos minerales aún no descubiertos, siempre sobre una concepción amplia, y de los modelos metalogenéticos y los condicionantes, tanto de su aparición o descubrimiento, como de las circunstancias prácticas de su aprovechamiento real y económico.

\section{Una panorámica del cobre}

Sería desde la Segunda Revolución Industrial con el empleo de la electricidad, cuando comenzara el uso del cobre a escala industrial, encontrándonos, entrado el siglo XXI, que su gran efectividad conductora le convierte en clave para el desarrollo de energías limpias, contribuyendo en la generación y empleo de la energía eléctrica de una manera más eficiente y menos contaminante, en sus redes de transporte o nuevos generadores, ayudando así en la mejora de eficiencia en parques eólicos, plantas fotovoltaicas, hidroeléctricas, así como en los motores de coches eléctricos (híbridos), etc.

El cobre, en valor, es el tercer metal en su producción mundial tras hierro y aluminio, cotizando en las bolsas internacionales (LME, NYME, Shanghai, etc.) junto con las principales materias primas, participando en precios spot, así como en el mercado de futuros, lo que da una idea de la importancia de este metal básico (Fig. 1).

Uno de los mejores indicadores del consumo de cobre en un país resulta ser, curiosamente, su renta per cápita (Fig. 2). Se considera habitualmente el grado de desarrollo de un país por el crecimiento de 


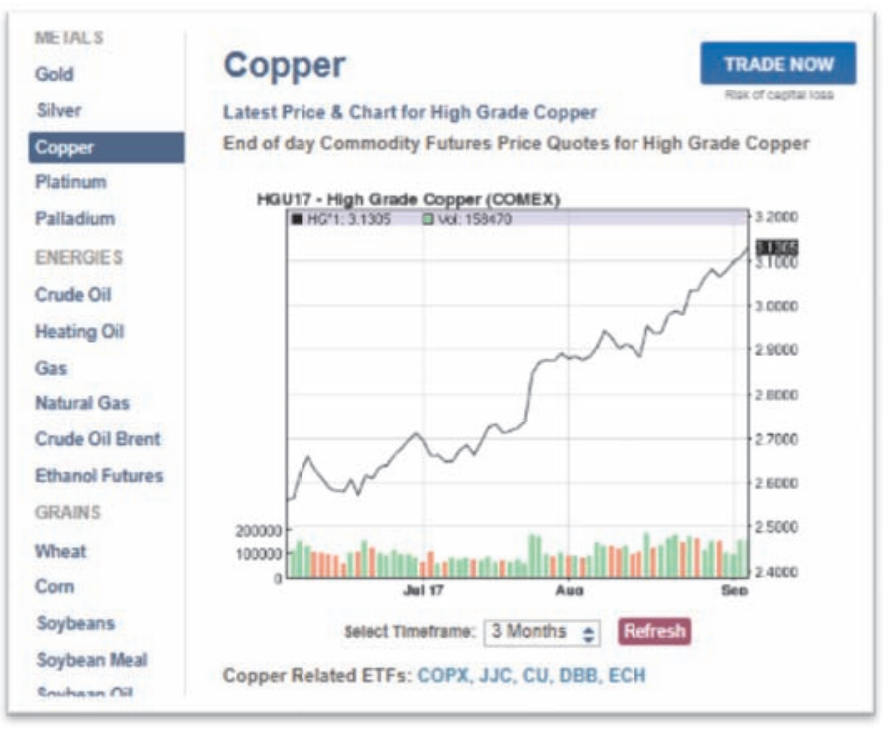

Figura 1. Bolsa de Materias Primas (http://www.nasdaq.com/markets/copper.aspx).

Figure 1. Raw Materials Exchange (http://www.nasdaq.com/markets/copper.aspx).

su PIB y, hasta estos días al menos (Chen S., 2007), su consumo eléctrico viene siendo una clara imagen del avance del mismo (una industria pujante es gran consumidor energético, una mayor urbanización o unas casas de mayor tamaño, también) (Kraft J., 1978); esto, sin entrar en debate con el concepto de la curva de Kuznets ambiental, sin embargo lo que sí parece claro es que, a pesar de que algunos autores debaten la desaparición de esta relación (Lin B., 2016; WoldeY., 2014), el hipotético camino hacia el desacoplamiento entre el consumo de recursos y el crecimiento, o las nuevas políticas de racionalización del consumo, en el caso del cobre deberán considerar la nueva llegada de la "tecnología verde" que potenciará el consumo de cobre en artículos como los vehículos eléctricos o las energías renovables (recuerda a la paradoja de Jevons: una mejora medioambiental que permite la reducción de los gases de efecto invernadero, implica mantener la extracción de minerales de cobre con una ley cada vez menor).

Es bien conocido que el empleo del cobre en la industria se debe principalmente a sus propiedades conductoras del calor y la electricidad, su maleabilidad, y su resistencia a la corrosión y a las altas temperaturas (aleaciones). Sin embargo, se debe señalar que es el conjunto de varias de éstas lo que le convierte en un material de difícil de sustitución, aun a pesar de las alternativas funcionalmente más específicas encontradas (p.e. minerales como el aluminio, buen conductor de la electricidad, pero con peores propiedades en el trefilado, o artificiales como la fibra óptica en las comunicaciones, gran conductor, pero de menor resistencia física, o los plásticos para conducciones de agua). Mencionar que más de la mitad de sus aplicaciones provienen del sector eléctrico, empleándose también en el sector de las telecomunicaciones, la construcción y la automoción.

Como nexo entre ideas sirve la observación de D. Bell, catedrático de sociología en Harvard, que al hilo de una pregunta sobre recursos naturales y tecnología respondía que "la mayor acumulación de cobre

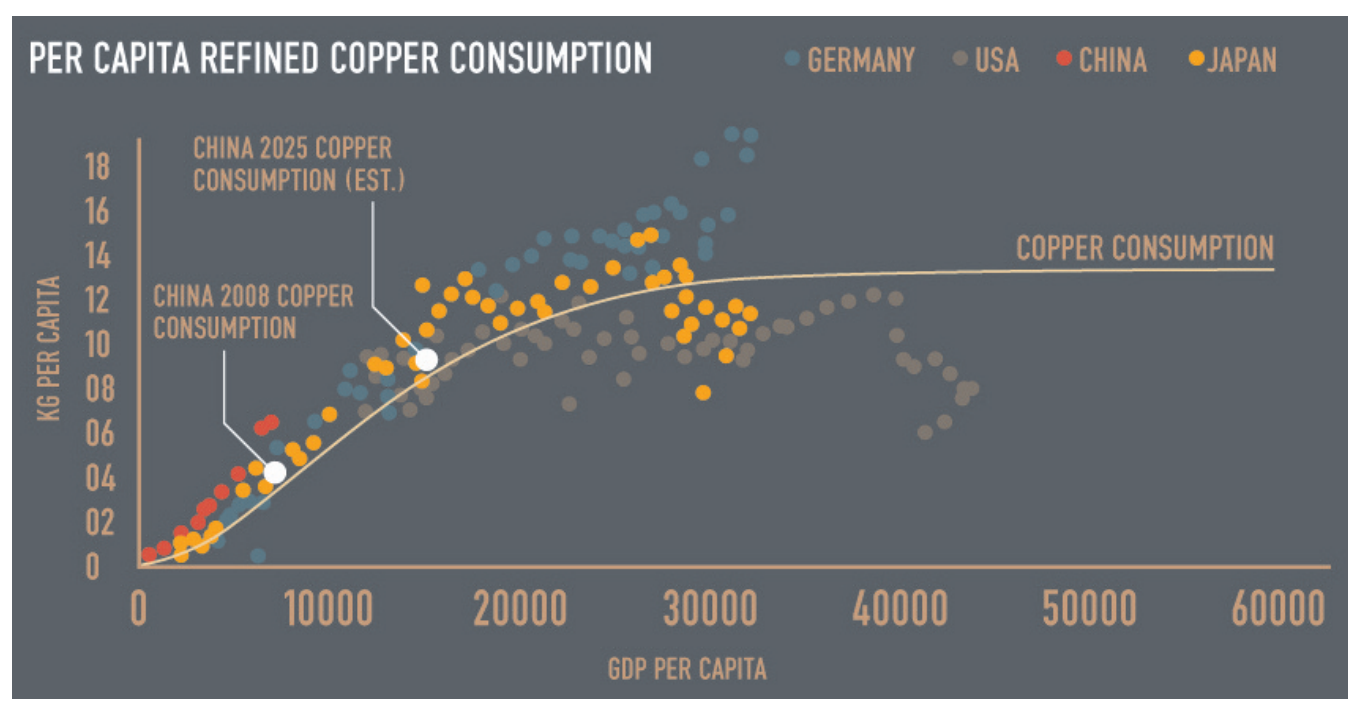

Figura 2. Consumo per capita de cobre vs. PIB (copperalliance.org.uk/images/ libraries provider3/copper-infographic-part-2.jpg). Figure 2. Copper per capita consumption vs. GDP. (copperalliance.org.uk/images/ libraries provider3/copper-infographic-part-2.jpg). 
en el mundo está bajo la ciudad de Nueva York, en forma de líneas telefónicas" (Krauze, E., 2016). Esta ubicuidad del metal rojo necesita, como se observa en el gráfico de la Fig. 3 y Tabla 1, de una producción de cobre en fuerte aumento (así se observa en los últimos años) y, aunque continúan los principales actores de su producción, van apareciendo cambios en la participación de los mismos. Si en 2005 Chile alcanzaba el $35 \%$ de la producción global del metal, en 2015 bajó al 30\%. En esos 10 años Chile subió un 8\%, mientras la de Perú creció $37 \%$, la de China $114 \%$, la de Zambia $72 \%$ y la del Congo, un $956 \%$.

El cobre suele reflejar los ciclos económicos con grandes fluctuaciones. Las variaciones en la demanda elevan o disminuyen los precios que, a su vez, incrementan o disminuyen una producción sujeta a elevados tiempos de maduración y fuertes inversiones.

Los altos precios pueden provocar inversiones en exploración, que con la llegada de nuevas explotaciones ven aumentado el suministro de cobre, lo que suaviza los precios y llena de nuevo los almacenes.

El precio del cobre en el mercado se considera un "output", no un "input" y, por tanto, marcado por la oferta y la demanda. Aunque los factores determinantes de estos precios son difíciles de conocer, sí se puede hacer una aproximación hacia algunos factores de su motivación, como pueden ser: el coste directo de producción unitaria o "cash cost"; el nivel de competencia en costes; el agotamiento de los principales yacimientos productivos; la disminución de las leyes medias de la explotación minera; la apari-

\begin{tabular}{|l|l|l|l|l|}
\hline \multicolumn{5}{|c|}{ Principales minas de cobre 2016} \\
\hline Chile & BHP & Escondida & 1148 & $10^{3} \mathrm{t}$ \\
\hline EEUU & Freeport & Morenci & 485 & $10^{3} \mathrm{t}$ \\
\hline Chile & Codelco & EI Teniente & 470 & $10^{3} \mathrm{t}$ \\
\hline Chile & Anglo A. & Collahuasi & 455 & $10^{3} \mathrm{t}$ \\
\hline Chile & Anglo A. & Los Bronces & 400 & $10^{3} \mathrm{t}$ \\
\hline
\end{tabular}

\begin{tabular}{|l|l|}
\hline \multicolumn{2}{|c|}{ Producción mundial de Cobre } \\
\hline 2016 & $19.4 \mathrm{Mt}$ \\
\hline 2015 & $19.1 \mathrm{Mt}$ \\
\hline 2014 & $18.7 \mathrm{Mt}$ \\
\hline 2013 & $18.3 \mathrm{Mt}$ \\
\hline 2012 & $16.9 \mathrm{Mt}$ \\
\hline
\end{tabular}

Tabla 1. Datos de producción mundial de cobre (elaboración propia).

Table 1. World copper production data (own elaboration).

ción de sustitutos (aluminio, fibra óptica, plásticos) o la llegada de nuevas aplicaciones (vehículo eléctrico, energías renovables); el desfase de los stocks del metal ante crecimientos bruscos de la economía mundial (varios años son necesarios para que un nuevo proyecto suponga la puesta de producto en mercado), o los incidentes sociales (huelgas en las explotaciones más importantes del mundo, como Escondida y Grasberg en los dos últimos años), casi dejando temporal y parcialmente el precio en manos de los productores. También hay que considerar la

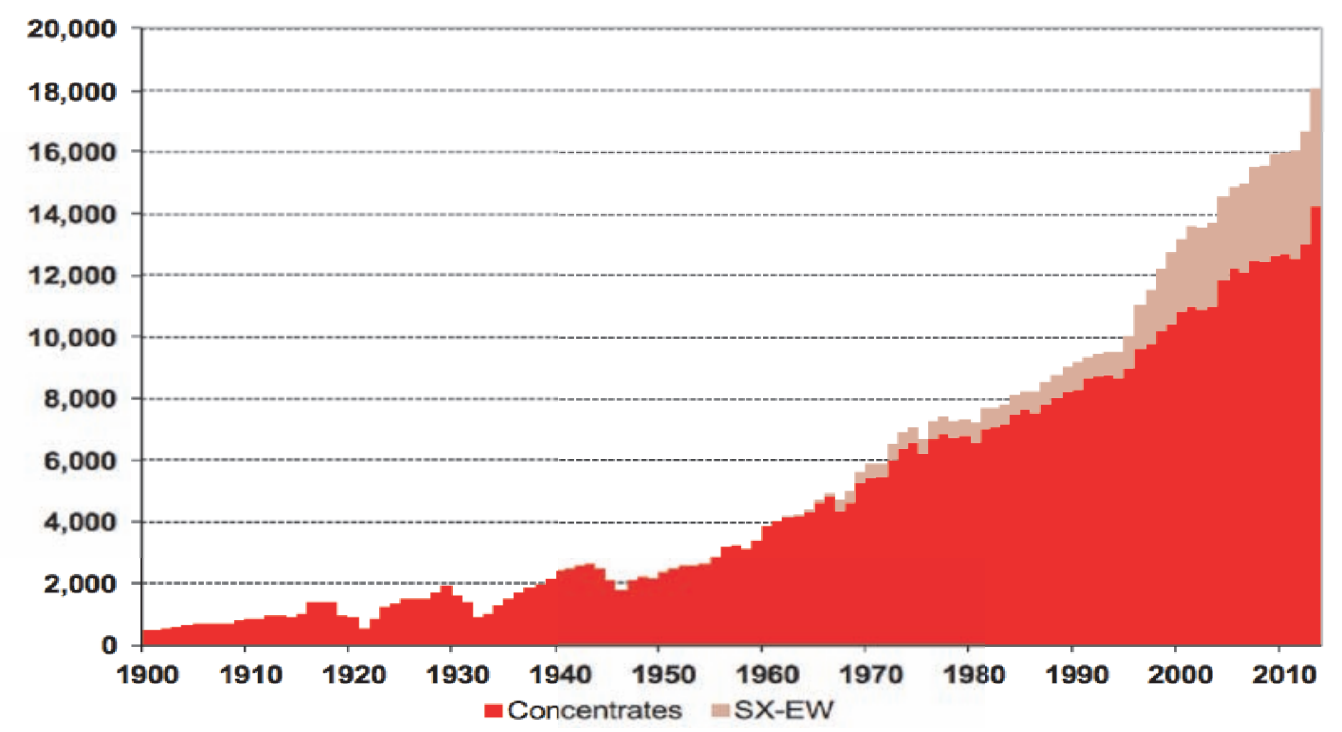

Figura 3. Producción mundial de cobre metal 1900-2013 (x1000t) (ICSG).

Figure 3. Worldwide production of metal copper 1900-2013 (x1000t) (ICSG). 
casuística habitual de los mercados en cuanto a la especulación, a la transparencia de la información respecto a las previsiones de escasez, y a la forma en que se presenta ésta ante los inversores.

El fuerte y continuado aumento de la demanda de materias primas en este comienzo de siglo $y$, por tanto, de su precio, ha llegado a definir ese período como el cuarto "super-ciclo" (Cuddington and Jerrett, 2008). Éste ha venido propiciado principalmente por la elevada demanda ante el crecimiento de países como China $y$, en menor término, India (con incrementos del PIB de $10.3 \%$ y $7.4 \%$ respectivamente en el período 2002-2012). Esto, principalmente, ha sido motivado por su rápida urbanización e industrialización, así como por un aumento del comercio exterior chino (conteniendo ambas un tercio de la población y un sexto del PIB mundial). El consumo de metales por parte de China se triplicó desde el 2000, alcanzando en 2014 el $47 \%$ del consumo mundial. Sin embargo, ese "super-ciclo" se ha detenido en los últimos años, al parecer, por una transición desde el reciente patrón de crecimiento intensivo chino en el uso de recursos, hacia una disminución en su industria pesada a favor del sector servicios.

Previo a la actual disminución en el crecimiento del gigante chino (PIB del 6\% anual estimado hasta 2020), el gran crecimiento en la demanda mineral de la primera década llevó a las principales empresas mineras a incrementar su producción mediante un aumento de capacidad a cualquier coste, con nefastas consecuencias ante la inesperada bajada de los precios en 2015, con caídas en el precio de las materias primas de un $25 \%$, provocando desplomes del $37 \%$ en la capitalización de estas grandes compañías (PWC, 2016).

En concreto, el precio medio en 2015 en el LME del cobre era de 5494 USD/t, y en 2014 de 6368 USD/t (una caída del 20\%) (ECl, 2015)

Dentro de una historia próxima, tras cinco años de estabilidad en los precios del cobre (en el entorno de los 1400 USD/t a 2000 USD/t), aparece el periodo 2003-2006 con fuertes subidas (de 4500 USD/t a 8778 USD/t) motivadas por un desequilibrio entre la oferta y la demanda. A finales de 2006 bajan los precios, ante la moderación de la demanda y la recuperación de la producción. La razón de fondo observada estriba en la variación en la demanda de países emergentes (solamente China participa con el $25 \%$ del consumo mundial en 2006 y un $47 \%$ del consumo metales en 2014).

La crisis financiera mundial de 2008 arrastró al consumo y los precios, remontando desde 2009 ante una economía china ávida de infraestructuras, hasta el año 2013, cuando vuelven a caer ante el cambio de modelo chino y la ralentización en su crecimiento. Desde junio 2016 aparece un repunte propiciado por la demanda sostenida de los países occidentales y las huelgas en dos de las principales minas del mundo. Las paradas en la producción de Escondida (Chile) y Cerro Verde (Perú), así como la decisión del gobierno indonesio en Grasberg (segundo productor de cobre del mundo) de introducir una nueva normativa en 2017 restringiendo las exportaciones de cobre, significaron una subida de precios del $11 \%$ (ver Fig. 4). (BM, 2017)

\section{El consumo y las reservas minerales}

Se ha observado que, en el último siglo, el volumen reconocido de cobre creció unas 25 veces (Schodde, 2010). Entre las razones de este enorme crecimiento
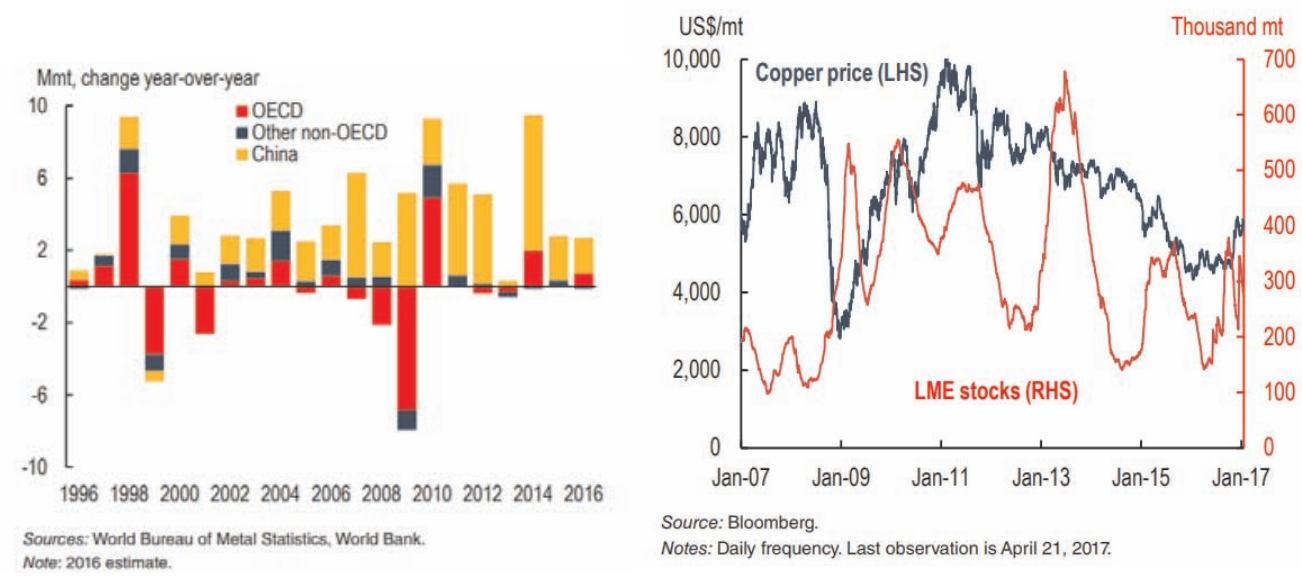

Figura 4. Consumo mundial de metales. Precios y almacenamiento por LME de cobre (Informe Trimestral del Banco Mundial, 2017). Figure 4. World consumption of metals. Copper LME Prices and Storage (World Bank Quarterly Report, 2017). 
en los recursos reconocidos se encuentran, aparte de una exploración exitosa y del precio como mecanismo natural de conversión recursos-reservas, la reducción de las leyes de corte o límites, la disminución de las leyes de mina en el transcurso del tiempo, o bien, la relación de la ley con el tonelaje, haciendo funcionar el mecanismo de validación recursos-reservas.

Respecto a la vida de las reservas, se observa que han descendido suavemente en los últimos sesenta años (Schodde, 2010) pero, aparte de los descubrimientos y los cambios en la ley límite, su velocidad de agotamiento viene influida por otras razones.

Para el estudio de la evolución de las reservas, aparte de intentar conocer en lo posible la oferta mineral, resulta apropiado analizar la evolución de su demanda. La realidad de la Curva de Kuznets (Fig. 5), ampliamente discutida, pero en vigencia, explica cómo en la incorporación de países al desarrollo pesa notablemente el consumo de recursos, momento tras el cual, da lugar al seguimiento de la $U$ invertida de su curva ambiental, encaminándose hacia consumos más contenidos. Ello, llevaría con el tiempo a una teórica disminución de la demanda de materias primas, una vez traspasado el punto de inflexión, disminuyendo así los ratios de consumo de reservas.

En este sentido, ya entrados en el siglo XXI, y con unas metas a perseguir hacia un desarrollo sostenible (Sustainable Development Goals, UNCTAD), se entiende como clave la disminución en el consumo de materias primas (reciclaje, eficiencia, economía circular, rediseño), pero sin sacrificar un progreso, a día de hoy asentado en el empleo de los recursos minerales (de laTorre, 2017). La idea del "decoupling" o disociación del ritmo del consumo frente al crecimiento económico, resultaría fundamental en los dos informes de la UNEP publicados sobre este tema.

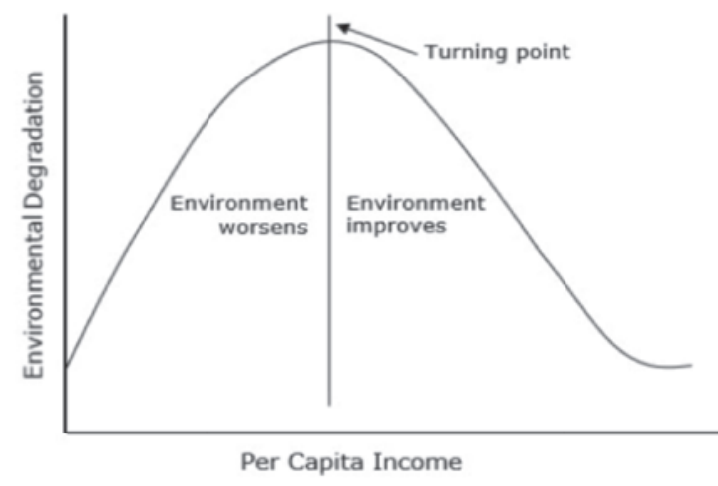

Figura 5. Curva de Kuznets ambiental. Figure 5. Environmental Kuznets curve.
El primer informe (Fischer-Kowalsky, UNEP, 2011) permitía pensar en un cierto desacoplamiento, al analizar cómo en el siglo XX se incrementaba por siete el ingreso global per cápita, mientras que el consumo medio de los recursos sólo se duplicaba. Sin embargo, el siguiente informe (Weizsäcker, UNEP, 2014) resultó más desalentador, reconociendo que el desacoplamiento, aunque fundamental, será difícil de alcanzar, y admitiendo que "el marco conceptual del desacoplamiento y la comprensión de los instrumentos para lograrlo están todavía en una etapa inicial" (Fletcher, 2017).

Lo que queda claro tras su lectura, es que, a pesar de los límites de los recursos naturales para soportar el desarrollo humano y el crecimiento económico, éstos seguirán siendo necesarios para la sociedad, por lo que las reservas en explotación continuarán descendiendo, en un panorama con un creciente número de habitantes que se espera alcance en los próximos años un mayor nivel de vida. En concreto, en el caso del cobre, existen previsiones que estiman un crecimiento en el consumo del cobre de aquí a 2035 de hasta un 43\%, en un escenario esperado de fuerte avance tecnológico (McKinsey Global Institute, 2017.)

Aunque los escenarios son cambiantes y la contabilidad de los recursos y reservas no es tarea sencilla, lo que no parece plantearse es la disminución del consumo de los metales en producción y, menos aún, en la extracción de cobre, mineral clave en la tecnología que apoya la nueva economía sostenible. Sería Fischer- Kowalsky quien señalara que «el progreso está relacionado con el aumento del metabolismo socioeconómico en diversos órdenes de magnitud" (Fischer-Kowalsky, 2000), lo que no fue óbice para que concluyera diciendo que, en aras de un desarrollo sostenible, habrá que mejorar la eficiencia material y energética, y efectivamente ese deberá ser el camino perseguido hacia el progreso.

\section{Las implicaciones del descenso en las leyes de cobre.}

La disminución de la ley mineral ha venido siendo patente, más que nunca, a lo largo del pasado siglo. La humanidad extrae primero los minerales allá donde resulta más óptima la operación y, por tanto, donde mayor calidad o ley descubre. Resulta así, que el cambio de condiciones (precio, tecnología, geopolítica) con el paso del tiempo es el que convierte en viables yacimientos que, por su bajo contenido en cobre, no lo fueron anteriormente. Algunas veces los nuevos escenarios permiten entrar a depósitos más ricos que la media, por haber estado paralizados en 
países en conflicto, porque la tecnología hubiera logrado abaratar costes, o bien, por permitir el beneficio de minerales antes marginales.

En la Fig. 6 se observa la disminución en las leyes de diferentes minerales (cobre, oro, plomo, zinc, níquel) en Australia desde 1840. Sirva como ejemplo, Australia, en la mina Burra en 1945, donde se alcanzaban leyes del $20 \%$ en cobre frente a las actualmente explotadas en ese mismo país de $0.87 \% \mathrm{Cu}$ en la mina Olympic Dam.

Para comprender la viabilidad de estos nuevos yacimientos de leyes bastante más inferiores, veremos más adelante cómo, la reducción de costes y la mayor accesibilidad, gracias a la tecnología, lo hacen posible. Se considera como ley mínima aceptable para la explotación de un proyecto en minería subterránea una ley media de $1.2-2.0 \% \mathrm{Cu}$, mientras que en cielo abierto ya se explotan habitualmente yacimientos de $0.5 \%$ Cu o algo menos (Ilegando hasta el $0.25 \%$ $\mathrm{Cu}$ cuando los metales acompañantes resultan interesantes, o bien se explotan óxidos de cobre).

Un caso de referencia es la explotación de pórfidos, que llegó a alcanzar a finales del siglo pasado el $62 \%$ de la producción de mundial de cobre, con una media del $0.44 \%$ de Cu (USGS 2008), estimando una disminución en ley desde el $0.49 \% \mathrm{Cu}$ de 2002 . En el USGS estiman que los factores origen de ese brusco descenso son la menor extracción de sulfuros de cobre supergénicos, una extracción de ambos tipos (sulfuros y óxidos) cada vez con menor ley mineral, el aumento en la minería de óxidos con menor ley, así como el aumento del precio del cobre, aumentando la cantidad de reservas que anteriormente no se consideraban económicas con menores leyes.

También, el principal productor mundial de cobre ha observado la fuerte disminución de sus leyes. Chile con un $30 \%$ de las reservas mundiales, sufre en su principal activo, la mina de Escondida con una producción del $9 \%$ del suministro mundial de cobre, un desplome en cuatro años que ha significado una bajada desde el 1.72\% Cu en 2007, hasta el 0.97\% Cu en 2011.

En la gráfica inferior (Fig. 7) se observa el decaimiento a nivel mundial de las leyes de cobre, que ha resultado constante desde principios del siglo $\mathrm{XX}$, momento en que comenzó el crecimiento imparable de la explotación de este mineral.

Un aspecto menos considerado al hablar de explotar leyes minerales más bajas, se refiere a cómo la ley afecta al consumo energético (Fig. 8), al estar relacionado con un mayor movimiento de tierras en la extracción y en su procesado. Aunque resulta crítica la fase de preparación mecánica de la concentración (la trituración y molienda son muy intensivas en energía), sin embargo, su metalurgia no se ve en exceso afectada por la ley inicial. El efecto de la ley en el SWB ("Solid Waste Burden") de la Fig. 9 muestra el incremento en la relación mena-ganga en función de unas leyes inferiores (Jahanshahi, 2009).

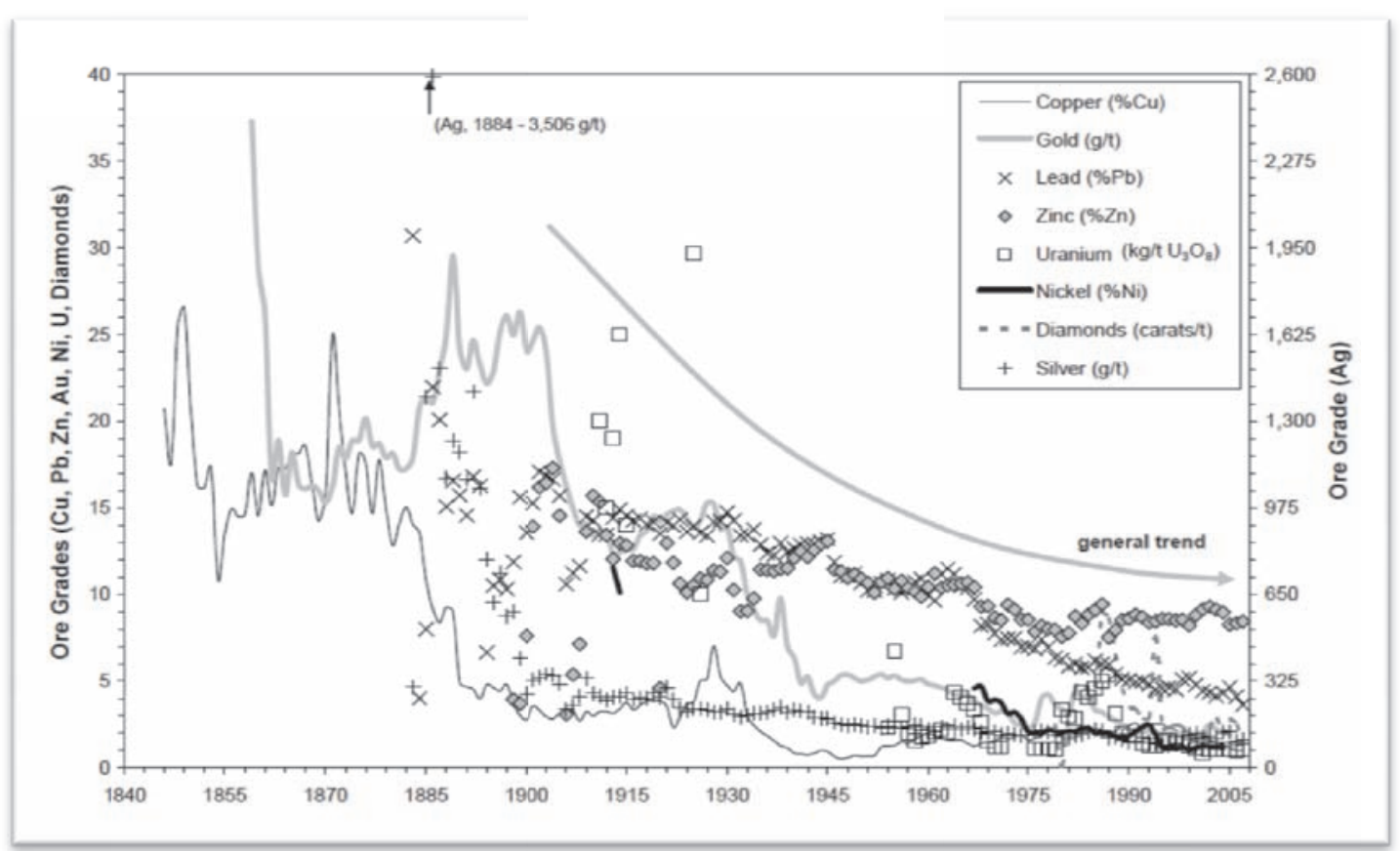

Figura 6. Disminución de las leyes minerales en Australia 1850-2005 (Mudd, G M, 2009, The Sustainability of Mining in Australia). Figure 6. Decrease in Australia ore grades 1850-2005 (Mudd, G M, 2009, The Sustainability of Mining in Australia). 


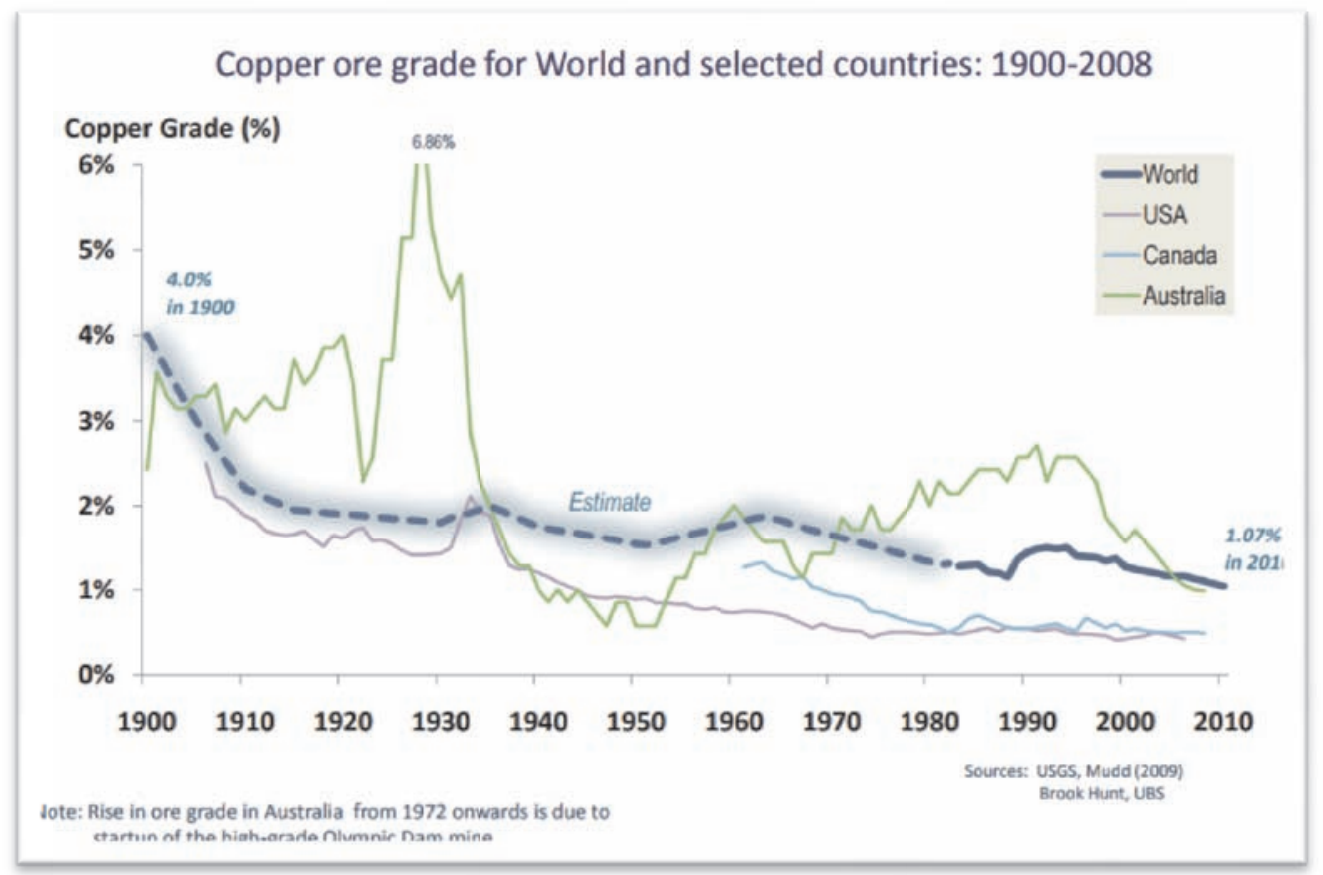

Figura 7. Descenso de las leyes de cobre 1900-2010. (Schodde R., 2010 MEMS Conference Mineral and Metal Markets over the Long Term). Figure 7. Decay of the copper ore grades 1900-2010. (Schodde R., 2010 MEMS Conference Mineral and Metal Markets over the Long Term.

Atendiendo al consumo energético, para el caso de la metalurgia extractiva aparecen dos posibilidades marcadas por el tipo de mineral. La vía más frecuente está marcada por la presencia de minerales de cobre sulfurado. Entre ellos, la calcopirita $\left(\mathrm{CuFeS}_{2}\right)$ que supone el $70 \%$ de las reservas de cobre conocidas, y se corresponde con la pirometalúrgica (horno/convertidor y electro refinado), implicando un menor consumo energético (33 $\mathrm{MJ} / \mathrm{kg}$ de GER), pero con el inconveniente medioambiental de producir $\mathrm{SO}_{2}$. La vía alternativa, la hidrometalurgia, se emplea habitualmente para el tratamiento de óxidos (menos comunes) mediante el proceso SX/EW, de menor impacto ambiental al contenerse mejor el $\mathrm{SO}_{2}$ como residuo líquido, aunque resultando la electrolisis intensiva en consumo eléctrico (64 MJ/kg de GER).

Todo esto puede llevar a pensar que, ante leyes medias inferiores, el agotamiento futuro de la tipolo-

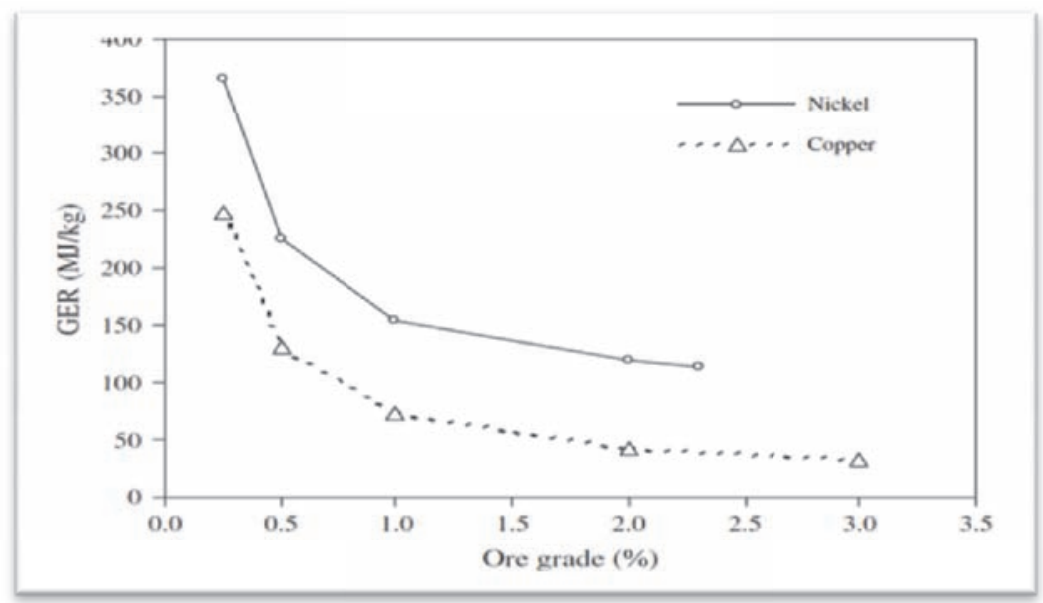

Figura 8. Efecto de la ley en el GER en la producción del cobre (UNSW Sydney William Rankin, 2016).

Figure 8. Effect of the ore grades on GER referring to copper production (UNSW Sydney William Rankin, 2016). 


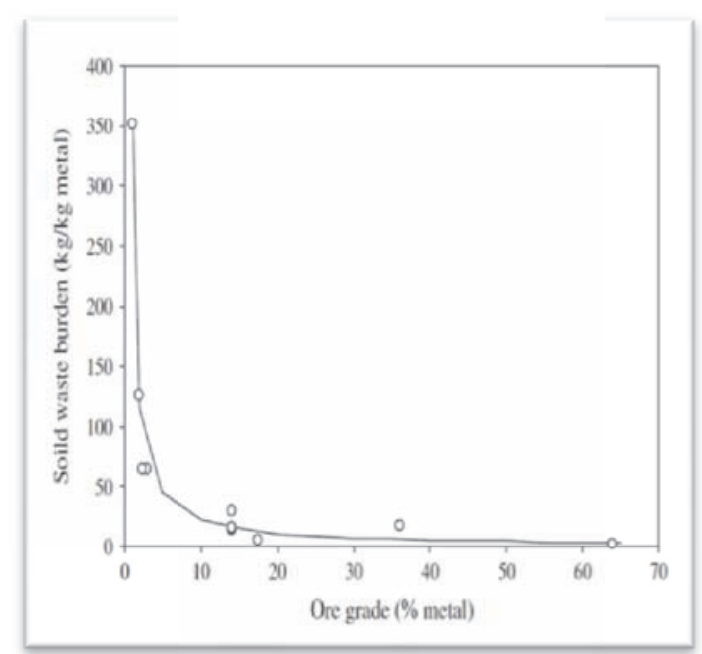

Figura 9. Relación entre la ley mineral y el material a vertedero. (UNSW Sydney William Rankin, 2016).

Figure 9. Relationship between ore grades and material to landfill. (UNSW Sydney William Rankin, 2016).

gía habitual de explotación del cobre, el pórfido cuprífero, podrá venir condicionado por la limitación medioambiental.

Así pues, la pregunta que debería plantearse la sociedad es si aceptaría el consecuente aumento en el consumo energético y de los inconvenientes ambientales por una menor ley. Contemplando únicamente el aspecto energético, esto significaría, en la obtención de cobre metal, un aumento de dos a siete veces el GER ("Gross Energy Requirement") actual para 2050, con un escenario ya descontado de energía únicamente renovable, y debiendo considerar los cambios tecnológicos, las futuras tasas de reciclado y la demanda eléctrica (Harmsen, 2013). Podría resultar que, en el medio plazo, la decisión de explotabilidad también se convirtiese en una evaluación social que pondere el reparto de beneficios ("Economical Parity") y la protección medioambiental, más que la escasez en sí misma.

Resultan bastante llamativos ciertos casos de gigantismo (actualmente se llegan a explotar leyes de 0.2-1.0\% Cu en los pórfidos, y más aún en otros metales como el $0.005-0.03 \%$ Mo, $0.004-0.35 \mathrm{~g} / \mathrm{t} \mathrm{Ag}$ y $0.2-$ $5.0 \mathrm{~g} / \mathrm{t} \mathrm{Au}$ ), donde una menor ley obliga a economías de escala que implican mayores ratios de desmonte y un incremento en los consumos energéticos, al igual que mayores cantidades de residuos, que marcarán el límite de la minería moderna.

El estudio del ciclo de vida o de su análisis exergético deberá conducir, en el caso del cobre, a la aparición de una minería subterránea que explote otras tipologías también económicas por sus mejores leyes.

\section{La curva de posición competitiva de costes}

Hoy, la abundancia de información lleva a considerar que el sector minero se mueve en una situación de casi transparencia económica. Una herramienta básica al alcance de todo el mundo son las curvas de coste de producción de la minería mundial, expresadas casi siempre por sustancias minerales o mejor, por metales específicos. De ellas se consideran los siguientes grupos:

1) Los Cash Cost o gastos directos de producción de la sustancia considerada, en forma de cantidad dólares americanos por unidad de producto vendible, según la unidad común de venta en el metal considerado (toneladas de metal puro o de concentrado de mineral vendible, onzas troy, libras y otros).

2) Los Total Cost que incluyen todo tipo de gasto implicado en la operación económica (incluyendo los generales y los relacionados con las inversiones).

3) Además, en los últimos tiempos se han popularizado las curvas de gastos sobre los llamados AISC (costes de producción totales más las inversiones de sostenimiento más los gastos generales y los gastos de exploración).

En todo caso, la aplicación de las curvas al mundo del cobre es muy completa y fácilmente accesible en distintos orígenes. De ellas se extraen las siguientes consecuencias.

Para los yacimientos de cobre mundiales, incluyendo todas sus tipologías y tamaños, unas leyes elevadas o depósitos cercanos a la superficie, hoy resultan muy difíciles de conseguir, en especial los depósitos con una baja razón de desmonte que permitirían disponer de unos bajos costos de operación.

Los depósitos más profundos requieren métodos de minería subterránea que, por lo general, significan mucho más altos costes de operación.

Conseguir un proyecto dentro del percentil 50 de la curva de costes (Fig. 10) es cada vez más complicado, debido a que los yacimientos conocidos con leyes más altas son generalmente más profundos y los superficiales todavía no desarrollados poseen leyes muy bajas (Fig. 7).

Existirán muy pocos proyectos que superen los obstáculos de, al menos, un 15\% TIR y, para la seguridad a largo plazo, que estén dentro del percentil 50 de la curva de costes, con la seguridad que esto supone ante bajadas de precio.

En cualquier caso, habrá un retraso considerable antes de que los proyectos que cumplan las condicio- 


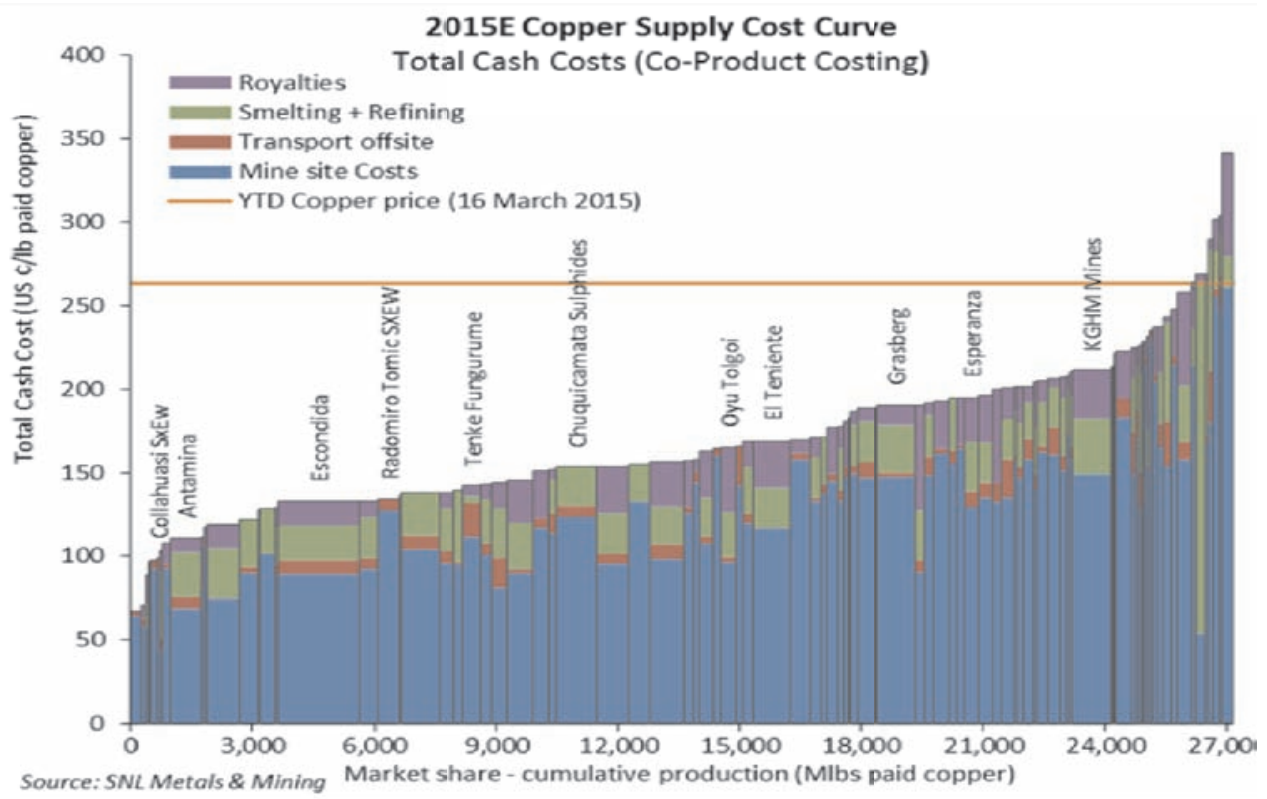

Figura 10. Cash Cost de las principales minas de cobre (SNL Metals\&Mining, 2015).

Figure 10. Cash Cost of the main copper mines (SNL Metals \& Mining, 2015).

nes anteriores estén listos para la producción, y este retraso resulta inevitable durante un tiempo, en el cual, el suministro del metal se reducirá y la demanda aumentará gradualmente o incluso de manera drástica. Esto conducirá a la siguiente subida del precio del metal. Lo único que es incierto en este escenario, tal como la historia ha registrado en varias ocasiones, es cuándo va a suceder.

Este mecanismo tiende a eliminar los proyectos con costes extremos superiores como consecuencia de las reducciones de precios cotizables, y contempla la eliminación de los proyectos de costes bajos por agotamiento acelerado o bien por sus reducidas dimensiones.

El logro de reducción en costes en estos últimos años para continuar manteniéndose dentro del percentil adecuado ha sido habitualmente originado gracias a las nuevas tecnologías aparecidas entre los años 1905-2007, logrando una reducción de costes de en ese período de hasta el $70 \%$ (ver Fig. 11). Otro parámetro que resulta significativo a la hora de reducir los costes de mina son las economías de escala, que en ese mismo período alcanzaron una mejora del $30 \%$.

A igual producción, las bajadas en ley implican mayores costes por mayor movimiento de tierras, tratamiento, y mayor energía, necesitando del gigantismo, o considerando la minería subterránea tras mayores leyes. Sin embargo, la experiencia actual en los costes de producción indica que los efectos en su reducción a causa de las nuevas tecnologías pueden más que su incremento debido al descenso en la calidad de los yacimientos.

\section{Teorías sobre la escasez mineral}

Reconocido por todos, el exponencial crecimiento en el uso de los bienes naturales como señas de identidad del pasado siglo $\mathrm{XX}$ y del que comenzamos recientemente, no resulta nada extraño el interés creciente en conocer la realidad sobre la escasez o la abundancia de los recursos no renovables. Resumimos a continuación algunos planteamientos de base utilizados en la aproximación a la respuesta de esta cuestión fundamental.

El enfoque del stock fijo/stock variable es fuente de debate a la hora de evaluar la capacidad de suministro mineral en el tiempo. El enfoque pesimista del "Stock Fijo" parte de unas reservas minerales fijas y determinadas, así como de una demanda creciente. De esta manera, la vida de las reservas de un mineral se calcula mediante el cociente entre las reservas conocidas de éste y su consumo, resultando cuanto menos ingenuo, al quedar de lado el potencial de la exploración y las nuevas tecnologías a la hora de contabilizar nuevas reservas (p.e. un fondo marino inicialmente con acceso a $700 \mathrm{Mt}$, posibilidad de explotación a mayor profundidad, nuevos procesos metalúrgicos SX/EW), la capacidad de reciclaje o los posibles sustitutos entre otros.

El enfoque más empleado, dada su mayor aproxi- 


\section{Key Technical Innovations}

Estimated average operating costs for copper mines in Western World: 1900-2009

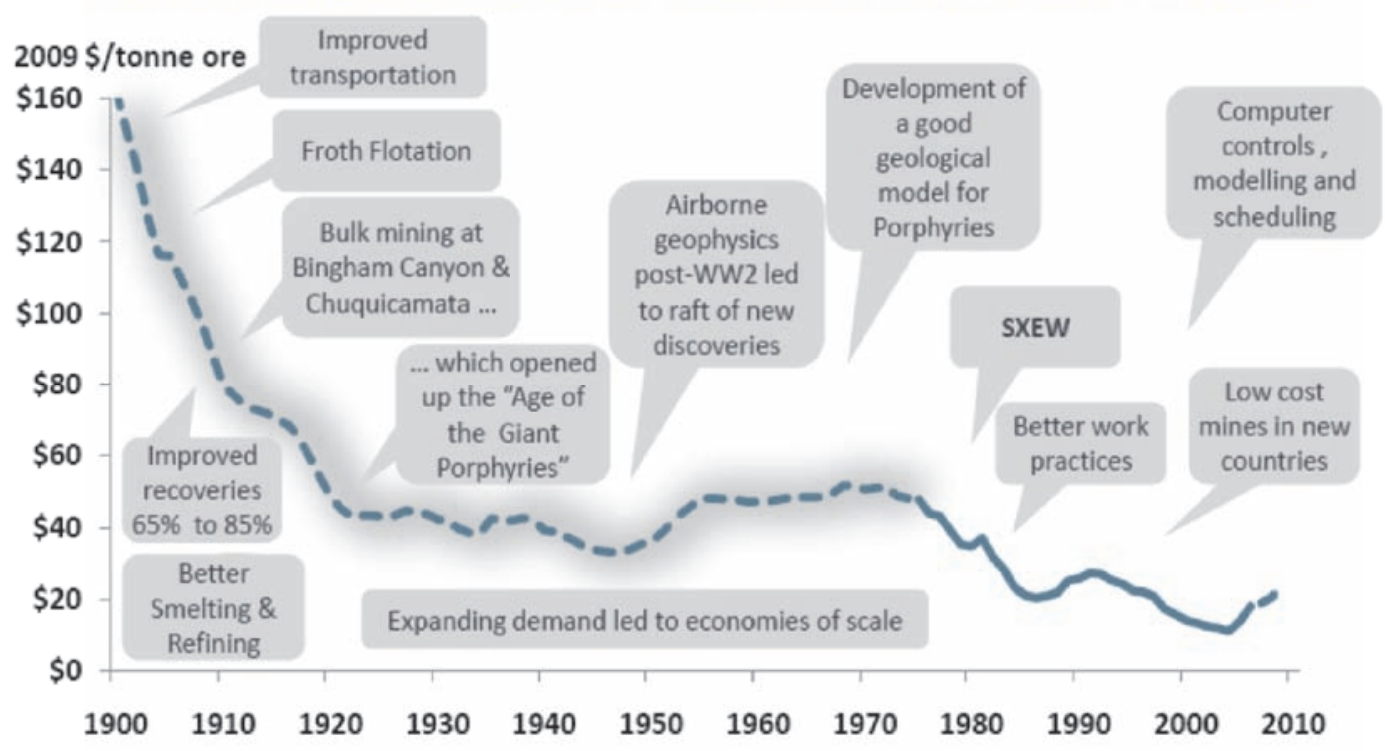

Figura 11. Principales innovaciones técnicas y su influencia en los costes de operación (Brook Hunt, CRU, Historical reports. MinEx Consulting estimates for 1900-1974).

Figure 11. Main technical innovations and their influence on operating costs (Brook Hunt, CRU, Historical reports. MinEx Consulting estimates for 1900-1974).

mación a la realidad, es el del paradigma del costeoportunidad: ¿Qué debe la sociedad abandonar para obtener otra tonelada de cobre?

Así se debería considerar, más que la existencia de un agotamiento físico, la teórica aparición de un agotamiento económico y gradual que alcanzaría un punto donde el coste de producir ese metal no resultara asumible. En economía, se considera un aumento en la escasez de un bien, cuando sube el precio del mismo en el mercado, por lo que, de nuevo económicamente, una disminución en el coste de producción de ese bien (factor influyente en la bajada del precio) alejará la escasez del mismo. Desde el pasado siglo, parece existir una carrera entre el aumento en los costes de extracción (provocado por menores leyes, ubicaciones más remotas o conflictivas, etc.) que, junto con el de los precios del metal (en aumento en función de su demanda), se va contrarrestando principalmente con el abaratamiento logrado ante la aplicación de nuevas tecnologías (y economías de escala).

Para hablar con propiedad de escasez mineral, se debe tener en cuenta la presencia del "límite" de los recursos minerales en sus tres vertientes:

Límite mineral: referido a la posibilidad geológica de que un elemento llegue a concentrarse en la corteza terrestre en manera de mineral. Su mineralogía propia y las tipologías de sus concentraciones son también elementos de discriminación económica.

2. El límite medioambiental es limitante. Nos movemos desde el entorno de "tres pilares" que deben conciliarse como desarrollo sostenible: el progreso económico, la justicia social y la preservación del medio.

La "vida de las reservas" calculada como reservas/consumo anual, para poder ser así considerada, debe contar con la esperada disponibilidad futura (entrada en explotación de diferentes modelos minerales, su tamaño, los costos de extracción y procesado, nivel de las cotizaciones de los metales, la intensidad de la exploración y, por supuesto, el desarrollo de las tecnologías). Es, por tanto, un sistema dinámico.

3. Límite real: el señalado por el mercado.

Parece, en efecto, que la oferta futura vendrá marcada por el precio del metal, pero considerando también la creciente importancia de la conciencia medioambiental como factor limitante.

Serán igualmente factores participantes la producción según la disponibilidad de metales en la natura- 
leza, la dificultad y el coste de exploración, o las condiciones socio-políticas del país donde se explote.

En función del enfoque empleado por diferentes autores, existen diversas estimaciones sobre la escasez del cobre. Northey (2014) considera que existen suficientes recursos de cobre identificados para mantener la producción minera de cobre, por lo menos durante los próximos veinte años. Kesler (2007) que, considerando el modelo espacio-tiempo aplicado a la situación geológica de los depósitos de cobre de tipo pórfido de manera vertical, con el fin de calcular la capacidad de la Tierra en depósitos minerales de cobre la futura minería, llega a la conclusión de que podría abastecernos durante 5500 años. EI USGS (2017) identifica recursos de cobre de 2100 millones de toneladas que, añadiendo los $3500 \mathrm{Mt}$ de cobre no descubiertos (en 11 regiones), garantizarían unos 280 años en un consumo anual de $20 \mathrm{Mt} / \mathrm{a}$, (extrapolación de información sobre depósitos conocidos en y cerca de la superficie, así como en las partes menos exploradas y más profundas de la sub-superficie). Gordon (2006), considera que la extracción acumulada de cobre ha estado creciendo más rápidamente que el descubrimiento de nuevos recursos, y afirma que las explotaciones acumuladas se acercan a los descubrimientos (la escasez que se avecina fomentará una transición de sustitución del cobre por otros más abundantes como el hierro o el aluminio).

Con otro acercamiento, Skinner (1976) se focaliza en los recursos "no descubiertos" y dice que en algunas partes del mundo los recursos minerales todavía no han sido explorados intensamente, ya que todavía existen sistemas políticos inestables, depósitos inaccesibles, o bien, por otras razones políticas y sociales. Sin embargo, existe otra razón más poderosa: muchos depósitos aun no descubiertos lo son por la cobertera de rocas jóvenes que impiden que los sistemas actuales puedan detectarlos.

Resolver este reto requiere una acumulación de esfuerzos de índole político y tecnológico muy importante. No resulta tarea sencilla, al aparecer incertidumbres en la demanda (incremento de población y evolución de sus necesidades) y en la oferta (cantidad mineral explotable a lo largo del tiempo, en función de los diferentes precios), así como una todavía incompleta internalización de costes (de difícil valoración para su consideración, con la aproximación pesimista de la restricción ante las culturas indígena, biodiversidad y otros bienes comunes).

Sin embargo, la casuística habitual nos permite ser optimistas, de igual forma que hizo CODELCO en 2012. En la Fig.12 se observa como la principal empresa chilena estima el futuro incremento en el consumo del cobre mundial.

Por tanto, las inquietudes mostradas en algunos foros sobre el agotamiento de los recursos minerales $y$, en concreto, del mineral de cobre, al menos en el medio plazo, se limitarían a ser una amenaza inexistente. Se aceptaría, en este sentido, hablar de un posible agotamiento del escenario económico, tecnológico o social, así como de sus condiciones, o de un progresivo menor acceso a alguna tipología concreta,

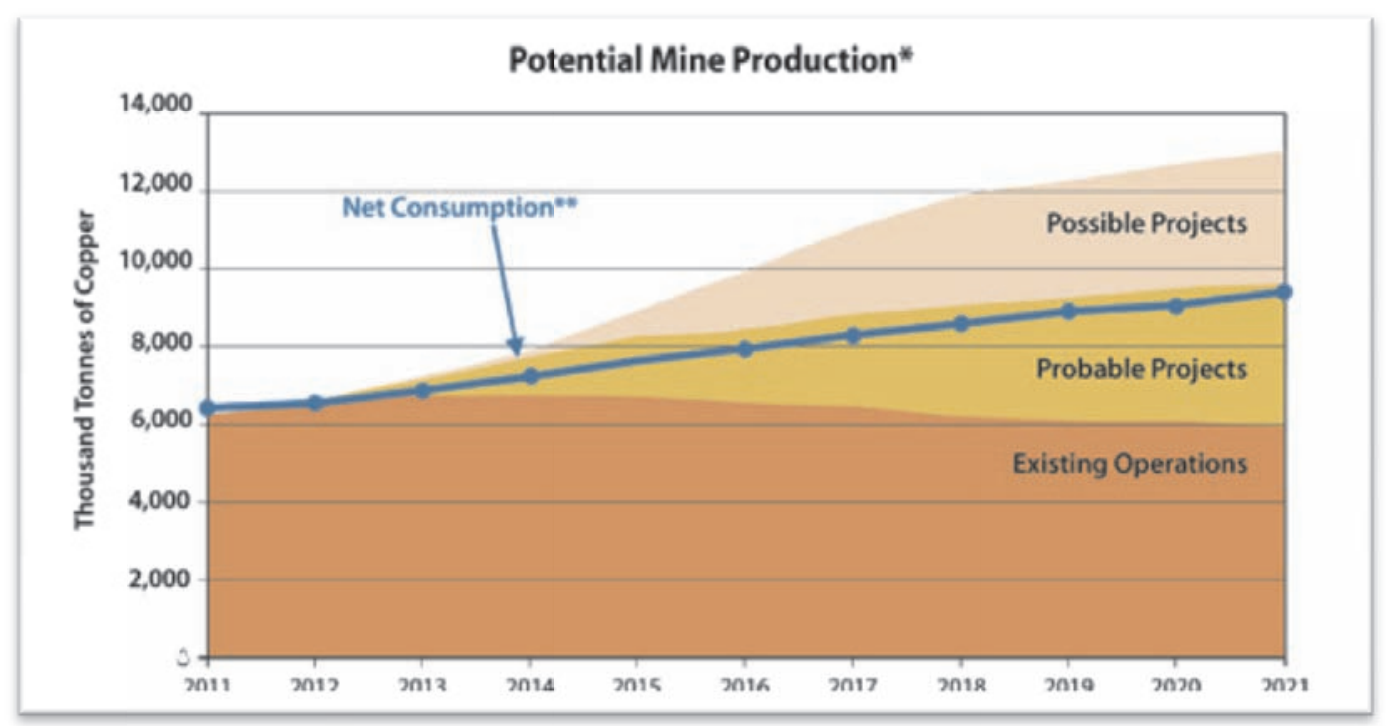

Figura 12. Estimación del potencial de producción de mineral de cobre. (Informe Codelco 2012).

Figure 12. Estimation of copper ore production potential. (Codelco Report 2012). 
pero no como agotamiento preocupante del mineral de cobre.

Sin embargo, resulta lógico aceptar la llegada del agotamiento de los yacimientos en operación, pero que, a su vez, serán sustituidos por descubrimientos nuevos o adyacentes (brownfield/greenfield). De igual forma, es ineludible que se produzca un incremento en los costes por la exploración de nuevos yacimientos en ubicaciones remotas, la paulatina disminución de las leyes de los yacimientos, mayores ratios de desmonte con un mayor movimiento de tierras, una mayor necesidad de explotación subterránea, una metalurgia más compleja, precios energéticos más elevados, etc., que convertirán al mineral de cobre en más escaso, pero únicamente desde una visión económica.

La escasez como peor escenario puede llegar a producirse, pero únicamente de forma temporal, causada en todo caso, por alguna particular desviación en el balance oferta-demanda, bien por una inapropiada gestión de stocks, bien por una inadecuada inversión en exploración o en desarrollo tecnológico (no es tema baladí una planificación desajustada, ya que un $5 \%$ de sobreproducción puede producir pánico en el mercado, con las distorsiones que ello provoca).

\section{Sobre los modelos metalogenéticos}

Hasta la aparición del trabajo de Phillip Crowson "The copper conundrum" en el año 2011, muy poco se había escrito sobre la base geológica y genética de la producción mundial de cobre. Crowson en su trabajo, explica como los principales yacimientos de cobre se agotan o sufren significativas reducciones a través de los tiempos, cuando se acaban las condiciones iniciales que los hicieron atractivos.

La tecnología actual ayuda a los tipos o modelos metalogénicos que mejor se adaptan a las nuevas exigencias de la industria minera, tal como es el gigantismo de las explotaciones, en un esfuerzo a superar en algunos momentos las bajas cotizaciones y aprovechando las facilidades que presentan tipologías extensas, como pueden ser los pórfidos cupríferos.

Crowson señala que, en el año 1975, los pórfidos suministraban ya el $34 \%$ del cobre del momento, habiendo llegado al $62 \%$ mundial en el año 1998. Sin embargo, en 2009 esta proporción había caído hasta el $55 \%$.

El autor anuncia una poderosa razón que explica el descenso de las leyes puede deberse, entre otras razones, al cambio en el tipo de los depósitos explo- tables. En el siglo XIX una parte importante del cobre procedía de los VMS (sulfuros volcánicos sedimentarios), de los Skarns, del cobre filoniano y de los $\mathrm{SH}$ (Sediment Hosted).

Con la caída de las explotaciones en África, Europa y el Este canadiense, estos tipos de depósitos han contado cada vez en menor proporción, aunque algunos parcialmente han resucitado. De esta manera, las nuevas minas de la región andina desplazaron el centro de la producción mundial de cobre mientras que ya, en 1988 , los pórfidos eran el $47 \%$ y el $62 \%$ de la producción mundial en 1998.

Se admite que siempre se explotan primero los recursos de mayor ley, los más accesibles y de mejor tratamiento. Además, los avances tecnológicos y la escala, logran superar a los descensos graduales y constantes de la ley, la accesibilidad y la complejidad metalúrgica.

Riotinto, en España, fue el mayor productor mundial en 1877 y explotaba con leyes del orden del $3 \%$ Cu entre 1883 y 1888, declinando las leyes hasta el $1.21 \% \mathrm{Cu}$ en 1925. Las leyes medias fluctuaron alrededor de un estrecho rango desde los últimos años de los 70 a los comienzos de la década de 2000. Sin embargo, a partir del año 2002 volvieron a caer de la misma forma que lo hicieron en los 70 .

Según Philip Crowson, el ritmo de extracción de un depósito se incrementa en tanto y cuanto las compañías buscan la economía de escala que repercutirá en las leyes límites del yacimiento. Además, el progreso económico y la subida de la productividad tienden a aprovechar yacimientos no tenidos en cuenta con anterioridad. Sin embargo, la inevitable subida de los costes de producción dirige esta actividad en una dirección opuesta.

\section{Las tipologías o modelos}

\section{El contexto general}

Crowson también afirma que esa tendencia de los pórfidos ha contribuido en los últimos años al descenso del rendimiento en la producción de cobre. Este autor nos dice que no existe evidencia en la tendencia del tamaño y ley de los nuevos pórfidos. Por ello, piensa que la bajada en la ley media de los yacimientos minados procede de la combinación de la desaparición de los otros tipos, no pórfidos (leyes elevadas respecto a los pórfidos) y su descenso en la ley (en los pórfidos cupríferos) por agotamiento.

Sin embargo, hay que considerar que la denominación "pórfido" trasciende a su origen metalogenético y es, además, un conjunto de características de la 
tecnología extractiva que le son comunes: escasas leyes de cobre, explotación predominantemente superficial, técnicas de explotación dimensionadas a enormes producciones, etc.

Hoy los esfuerzos por encontrar nuevas tipologías de yacimientos de cobre son evidentes: aunque predominantemente subterráneas, los skarns, los VMS y, sobre todo, los SH (Sediment Hosted) e IOGC que parecen resurgir como respuesta a las causas explicadas.

Existen otros factores: el acceso a nuevos territorios, las presiones ambientales y sociales y el descenso relativo en los costes de explotación subterránea. No parece que las leyes de producción de un yacimiento se encuentren en total dependencia total con los precios de mercado, ya que resulta bastante complicado cambiar configuraciones de explotación en la mina $y$, sobre todo, necesitando un capital extra que, a corto plazo, no resulta fácil de conseguir.

También existen dos tendencias cuando caen los precios del metal, o bien cuando suben, optando en cada caso (en altos precios de mercado) por explotar con prioridad las leyes altas o más bien lo contrario (caso de Phelps Dodge y Codelco en la crisis del 97).

Los pórfidos cupríferos hasta hace pocos años se caracterizaban por sus enormes volúmenes de recursos (miles de millones de toneladas en algunos yacimientos) de los cuales solo se consideraba una pequeña parte de mayor y más fácil acceso y con un desmonte muy moderado. Su ley actual había sido una consecuencia de la desaparición de una parte de sus mejores concentraciones. Este proceso ha sido paulatino, pero también inexorable, tan solo modificado por la masiva explotación de minerales secundarios consecuencia de las innovaciones introducidas en el proceso de SX/EW.

Siempre existen excepciones, incluso la explotación de recursos existentes a cierta profundidad, sobrepasando ampliamente una mínima razón de desmonte compatible con un cut-off o ley de corte, reducido, a causa de los bajos costes derivados del gigantismo de las explotaciones.

\section{Las tipologías o modelos de los yacimientos de cobre}

La formulación de los modelos metalogenéticos nace de la necesidad de una comprensión del fenómeno de su creación con vistas a facilitar nuevos descubrimientos. Un aspecto de la expresión de los diferentes tipos de yacimientos es su asociación con series particulares de las rocas que los albergan (Stanton, 1972).

Esta asociación refleja ampliamente tanto el entor- no geológico y como los procesos que han contribuido a la formación del depósito. Las asociaciones identificadas se han utilizado ampliamente en la exploración minera.

Los geólogos, generalmente prefieren los sistemas de clasificación genética que también incorporan elementos de su composición, forma y asociaciones minerales. Sin embargo, el sentido del modelo viene a reflejar, no solo la génesis del depósito, sino que también asume su sentido económico, técnico y productivo. Así, como ejemplo, para un pórfido de cobre, al aceptar el término, también se asumen sus condiciones genéricas de muy bajo ratio de desmonte, forma más o menos esferoide, ley baja, conocimiento o dominio metalúrgico de su concentración, intervalo de riqueza sus concentrados y varios aspectos técnicos más.

\section{Características e implicaciones de los principales modelos}

\section{a. Los pórfidos cupríferos}

Los pórfidos de cobre son el resultado de las complejas interacciones de muchos procesos genéticos. Un depósito de pórfido de cobre se define por la existencia de los siguientes fenómenos geológicos:

1. Presencia de sulfuros de cobre que se localizan en una red de venillas o "stockwork" y como diseminación de granos en la matriz de la roca;

2. Alteración y mineralización en profundidad hasta 1-4 kilómetros, genéticamente relacionada con depósitos de magma emplazados bajo la corteza (6-8 km), de composición de intermedia a silícea de arcos magmáticos y encima de las zonas de subducción;

3. Complejos intrusivos que se emplazaron en el momento anterior a la formación de los depósitos de pórfido. Predominio de geometrías en forma de stocks de forma cilíndrica y vertical;

4. Áreas con superposición de alteraciones marginales propilíticas y fílico-arcillosas, y rodeadas por un conjunto de alteración potásica;

5. Cobre introducido durante los sucesos de alteración fílica-arcillosa.

En relación con la relación modelo-tecnología de explotación, la morfología de tabular a esférica junto (a veces) sus enormes dimensiones $y$, actualmente, su baja ley, son las características fundamentales que influyen en el aprovechamiento de los pórfidos de cobre.

Los bajos costes de producción son consecuencia del uso de maquinaria de grandes dimensiones $y$, 
sobre todo, por los bajos ratios de desmonte. Además, la intensa explotación acaecida en los últimos años ha reducido sustancialmente su ley y no tanto el tonelaje que albergan. De hecho, ya han comenzado sus adaptaciones: minería subterránea de alta productividad y bajo coste, minería subterránea en zonas de alta ley, intensidad productiva con minerales de baja ley y otros.

\section{b. Los skarn de cobre}

Los skarns son rocas metamórficas de grano grueso compuestas por minerales silicatados que se forman por sustitución de rocas carbonatadas durante el contacto o metamorfismo de contacto o regional.

La mayoría de los grandes depósitos de skarn del mundo están relacionados con los sistemas hidrotermales (Fig. 13) Los skarns son depósitos minerales de una relativa alta temperatura y relacionados con la actividad magmática-hidrotermal asociada con plutones graníticos en ambientes orogénicos.

Los skarn suelen ser más pequeños que los pórfidos, pero, de mayor concentración metálica. Puede decirse que los skarns se forman cuando un plutón ácido invade series sedimentarias que contienen episodios carbonatados.

Los principales procesos involucrados en la génesis: (1) el metamorfismo de contacto durante el

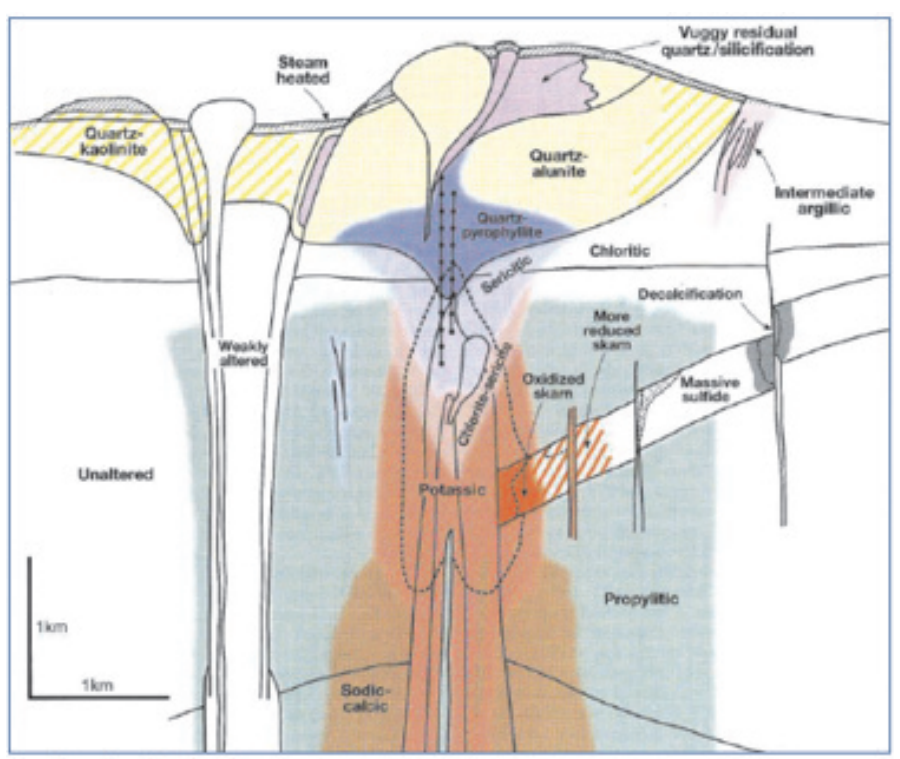

Figura 13. Zonas de alteraciones en un pórfido cuprífero y su relación con un "skarn" según Sillitoe (2010).

Figure 13. Alteration Zonality in a porphyry copper and its relation with a "skarn" type according to Sillitoe (2010). emplazamiento del plutón, (2) la formación metasomática progradante cuando el plutón se enfría y se desarrollan fluidos mineralizadores, y (3) la alteración retrógrada de las asociaciones anteriores.

En relación con el modelo-tecnología de explotación, la mayor parte de los minerales económicos de skarn están presentes como exo-skarn, formando una mineralización en las rocas carbonatadas que la aloja.

Los yacimientos de cobre tipo skarn son menos abundantes que los pórfidos del mismo metal, son más difíciles de descubrir y suelen ocupar posiciones no accesibles al reconocimiento superficial y directo. Suelen ser más ricos (en parte porque no han sido descubiertos con anterioridad), son más irregulares $y$, a veces, de difícil predicción, tanto geométrica como de sus leyes.

La mayoría de los nuevos yacimientos son de explotación subterránea y su importancia no decaerá en el futuro, siempre apoyados en la calidad unitaria o concentración de metal. Son difíciles de valorar y su planificación en mina requiere de mucha experiencia. La dilución en la explotación debido a su complejidad geométrica es alta y requiere un reconocimiento previo, más detallado que en los pórfidos a los que se encuentran relacionados.

\section{c. Yacimientos Volcánico-Sedimentarios (VMS)}

Corresponden a cuerpos estratiformes de sulfuros en unidades volcánicas o en interfases volcánico-sedimentarias depositadas originalmente en fondos oceánicos.

Como idea general, los VMS consisten en un $90 \%$ en pirita masiva, y contienen cantidades variables de $\mathrm{Cu}, \mathrm{Pb}, \mathrm{Zn}, \mathrm{Ba}, \mathrm{Au}$ y Ag. Los VMS se presentan en grupos y en áreas específicas y están restringidos a un número limitado de niveles estratigráficos.

Existe una asociación con rocas volcanoclásticas. Debajo de los depósitos de sulfuros normalmente existe un stockwork de venillas de sulfuros en rocas intensamente alteradas, que ha sido el alimentador de los fluidos hidrotermales. El stockwork en ocasiones puede poseer leyes económicas.

Estos depósitos están formados por emanaciones de fluidos hidrotermales asociadas al volcanismo submarino y se trata de depósitos singenéticos con la actividad volcánica submarina a la que se asocian. Se puden dividir de manera general entre los cercanos al centro efusivo (proximales) y los más o menos lejanos (distales) Fig. 14.

La mineralogía corresponde a una mezcla de sulfuros metálicos dominados por pirita y/o pirrotina con cantidades variables de calcopirita, esfalerita y 


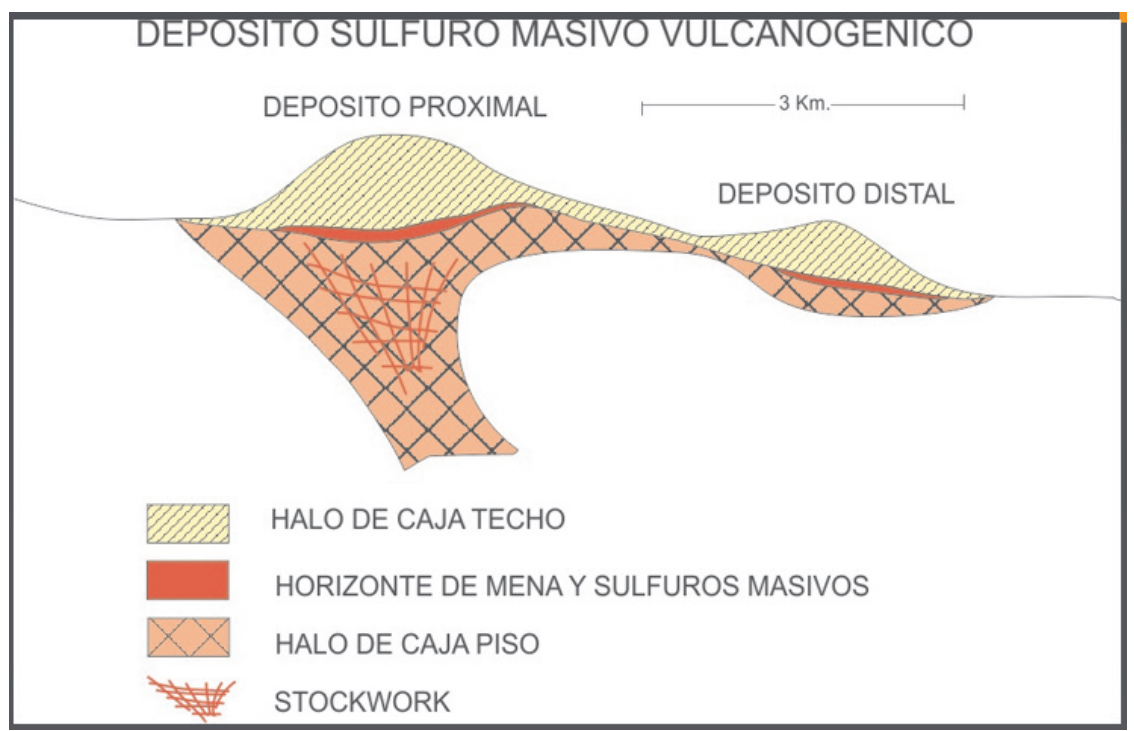

Figura 14. Esquema de los cuerpos principales de un VMS. Paulina Durán. 2015.

Figure 14. Scheme of the main bodies in a VMS ore deposit. Paulina Durán. 2015.

galena. La galena y esfalerita se ubican en la mitad superior de los depósitos, mientras que la calcopirita se concentra en la porción inferior y grada hacia abajo a un stockwork de venillas. Todo ello se puede apreciar en el esquema de zonalidad-alteración de la Figura 15.

Relación modelo-tecnología de explotación: La mayoría de los depósitos de sulfuros masivos del

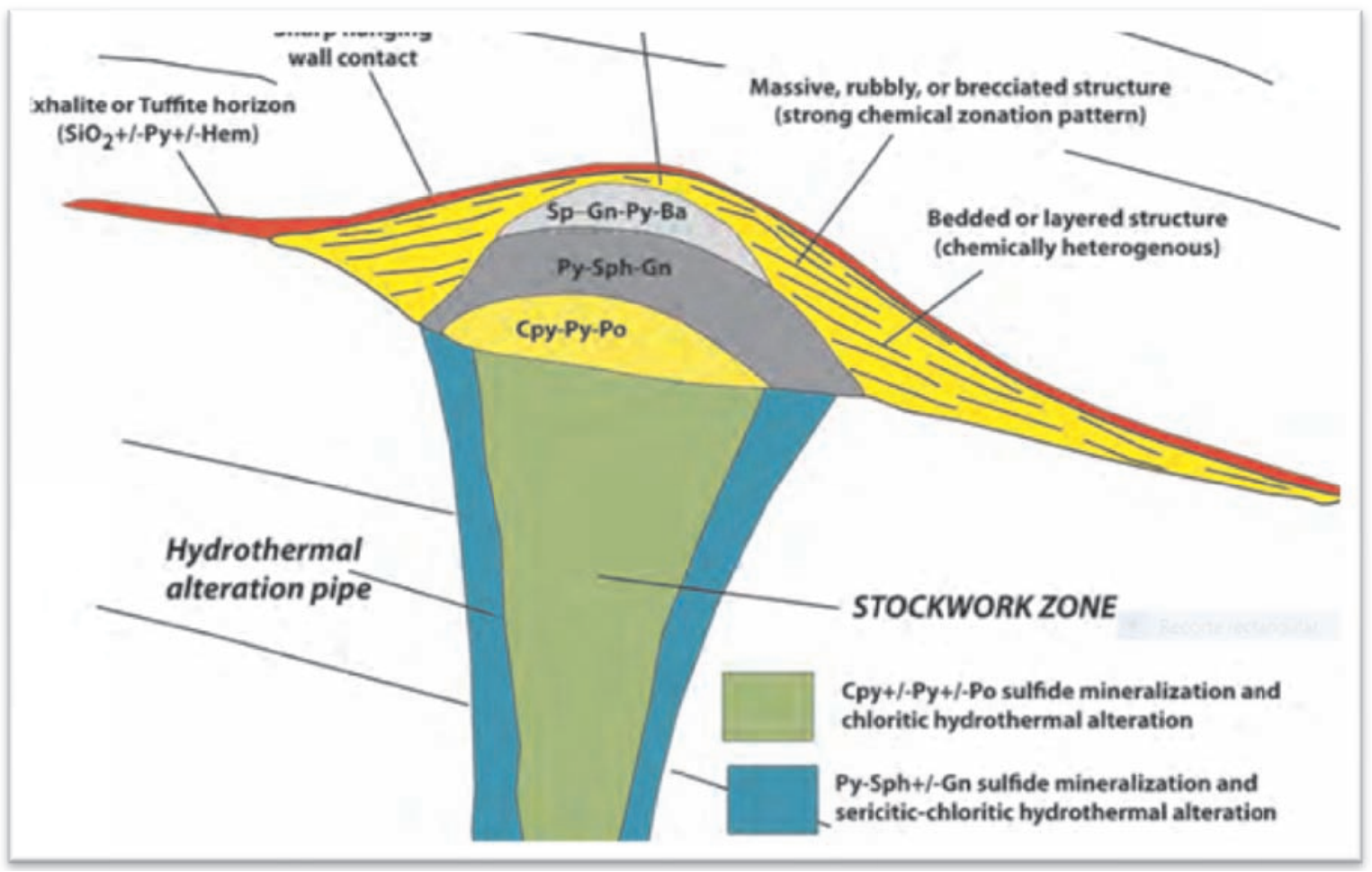

Figura 15. Zonificación fundamental de un VMS según Lydon 1984.

Figure 15. Fundamental zoning of a VMS according to Lydon 1984. 
mundo son relativamente pequeños y el $80 \%$ de los depósitos conocidos está en el rango de $0.1 \mathrm{Mt}$ a 10 Mt. De estos, la mitad contiene $<1 \mathrm{Mt}$ de mineral. Sin embargo, estos depósitos también pueden ser relativamente grandes o de alta ley. Se explotan distritos en que existen numerosos cuerpos mineralizados formando grupos.

Las riquezas iniciales han acabado con la existencia de los yacimientos superficiales. Hoy, la mayoría de los proyectos VMS son subterráneos y a veces selectivos. Al encontrarse en áreas plegadas es frecuente hallarlos en posición verticalizada. El método de "cut and fill" es el que predomina en yacimientos pequeños o medianos. A veces las grandes cámaras también son posibles.

La mineralogía variada y, sobre todo, el inter-crecimiento a tamaños de grano muy fino y la presencia de metales no deseados, es otra dificultad de su aprovechamiento. La abundancia de sulfuros es un reto para mantener el medio sin generar aguas ácidas.

\section{d. Yacimientos de cobre en series sedimentarias}

(SH)

Se encuentran estratoligados, es decir, están restringidos a un estrecho rango de capas dentro de una secuencia sedimentaria. Son epigenéticos y diagenéticos, formándose después de la deposición del sedimento huésped, antes de su litificación. Las deformaciones tectónicas posteriores pueden condicionar su disposición final como se aprecia en la Figura 16.

Los tipos de rocas huésped son dos: calizas o sedimentos dolomíticos de baja energía, pizarras y rocas carbonatadas de origen marino o lacustre; y areniscas de alta energía, arcosas y conglomerados de origen continental.
Existen dos tipos principales: de "facies reducidas con cobre" y "Red-beds" con cobre. Las rocas proterozoicas superiores $y$, sobre todo, las rocas neoproterozoicas son las más productivas. Las rocas del Pérmico en Europa y las rocas carboníferas inferiores en Asia Central son menos importantes. Otros pequeños depósitos se encuentran en el Phanerozoico, como en España.

El ambiente deposicional se relaciona con sedimentos altamente permeables en cuencas epicontinentales poco profundas y cerca del paleoecuador. Puede existir una zonalidad mineralógica y metálica ligada a los ambientes de deposición primaria en la cuenca, tal como se muestra en la Figura 17. Es habitual su relación con sabkhas y en sedimentos con alta tasa de evaporación, pero la configuración fundamental es tectónica. Los ambientes favorables son los relacionados con los rifts intracontinentales, los brazos fallidos de las uniones triples en aperturas intracontinentales y los márgenes continentales pasivos.

Relación modelo-tecnología de explotación: Los yacimientos de cobre tipo "Sediment Hosted" habían sido en parte olvidados debido a las condiciones geopolíticas de su aprovechamiento. Las potencias discretas de los $\mathrm{SH}$ se traducen en su explotación predominantemente subterránea. La buena definición de sus hastiales (baja dilución en el arranque), la continuidad de sus cuerpos mineralizados $y$, sobre todo, su ley media, ayudan a su explotación. Por su geometría variable será necesario el cambio de sistema de explotación, pasando de "Cámaras y Pilares con relleno" a "Subniveles" cuando se verticalizan.

Su mineralogía, con presencia de sulfuros más ricos en cobre que la calcopirita, produce concentrados de alta ley relativa en cobre, con lo que mejora el NSR del concentrado del yacimiento. La presencia de

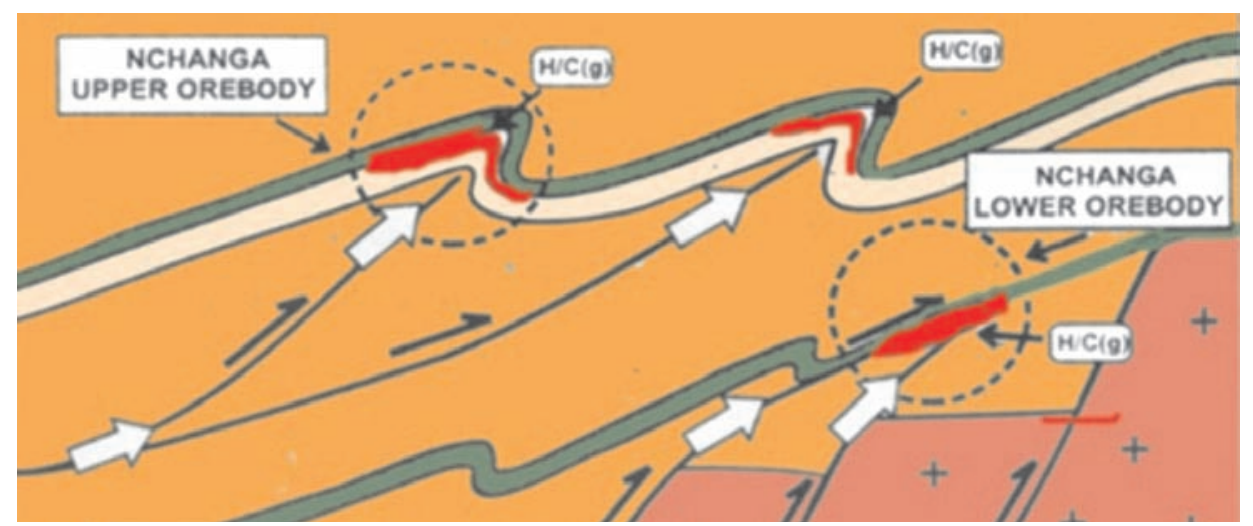

Figura 16. Controles estructurales en la mina congoleña de Nchanga. Tipo SH según McGowan R.R et al.

Figure 16. Structural controls at the Nchanga Congolese mine. Sediment Hosted type. McGowan R.R et al. 


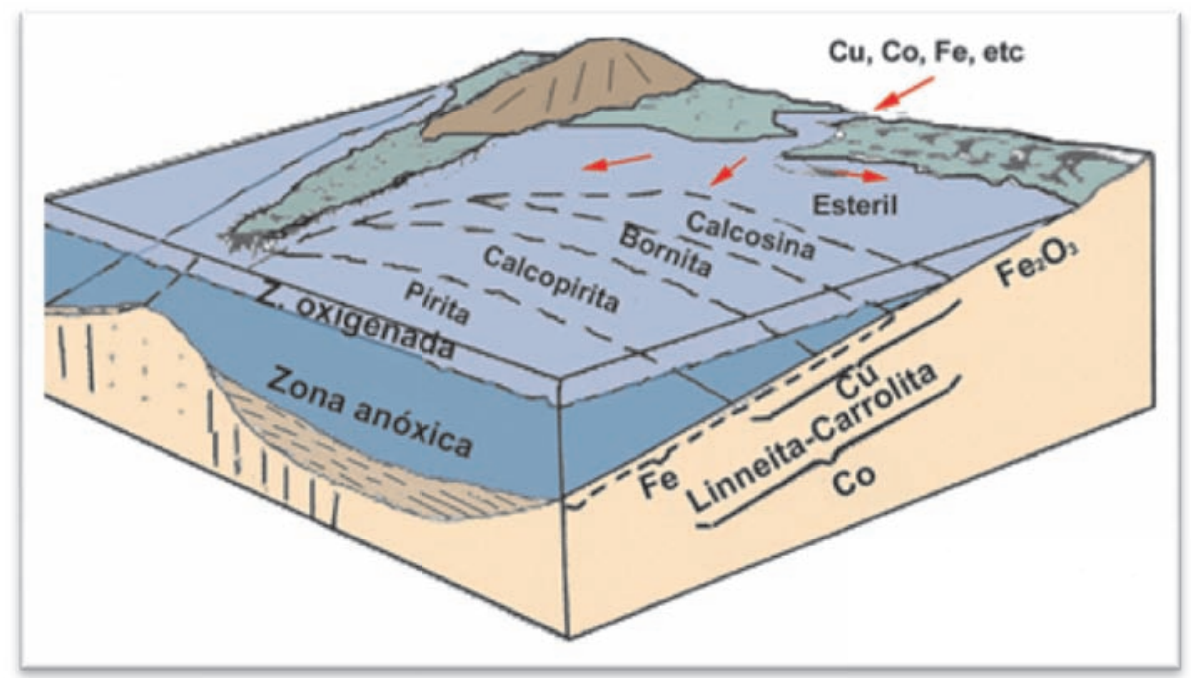

Figura 17. Zonalidad en el origen de un yacimiento SH (Sediment Hosted) según F. Vázquez (2012).

Figure 17. Zonality at the origin in a SH (Sediment Hosted type) F. Vázquez 2012.

otros metales de alto valor unitario, sobre todo el cobalto y la plata, ayudan casi siempre en los resultados económicos de su explotación.

\section{e. Los IOCG}

Son depósitos de óxido de hierro Cu-Au en forma de vetas y cuerpos de hematites y/o magnetita alojados en brechas con minerales diseminados de $\mathrm{Cu}, \mathrm{Au}, \mathrm{Ag}$, $\mathrm{Pd}, \mathrm{Pt}, \mathrm{Ni}, \mathrm{U}$ y Tierras Raras.

Esta tipología de yacimientos se forma en cuencas sedimentarias o volcano-sedimentarias intruídas por rocas ígneas. Los depósitos minerales se asocian de manera amplia con situaciones redox y se caracterizan por la alteración sódica de las rocas fuente y la alteración potásica de las rocas hospedantes. El enorme tamaño, la metalurgia relativamente simple y las altas leyes son características de este modelo que se traduce en explotaciones, a veces muy rentables.

También, los depósitos de IOCG se distinguen por su mineralogía. Los minerales más comunes son la calcopirita, formando 10-15 por ciento de la masa de roca. El mineral de la ganga es, típicamente, la hematites. Estos depósitos son principalmente de cobre de anfitrión, sin embargo, pueden o no contener niveles económicos del oro.

Relación modelo-tecnología de explotación: EI tonelaje de estos yacimientos varía ampliamente desde más de 3.800 millones de toneladas en Olympic Dam en Australia hasta los 470 millones de toneladas en La Candelaria, Chile. Dentro de los 33 depósitos de IOCG del USGS se pueden reconocer dos poblaciones de leyes y de tonelaje: 12 en depósitos alojados en venas y 21 en brechas o zonas de fallas.

La diferencia en el tonelaje (mediano $3.5 \mathrm{Mt}$ a 120 Mt entre los primeros y los segundos) refleja el tipo y la extensión de la permeabilidad en la roca huésped y la cantidad de espacio abierto disponible para la deposición de minerales. La explotación de sus variedades de mayor tamaño admite la explotación subterránea de gran tonelaje apoyada en sus notables leyes de cobre.

El aprovechamiento de otros minerales acompañantes también es otra característica. Así, Olympic Dam no solo es una principal productora de cobre sino una de las mayores concentraciones de uranio del mundo. La presencia de oro también es otro factor recurrente en muchos yacimientos de este tipo. Además del mineral de hierro, sobre todo de la magnetita, resulta una buena ayuda para la economía de estos depósitos.

\section{f. Cobre magmático}

Los depósitos de sulfuros magmáticos de $\mathrm{Ni}-\mathrm{Cu}$ se producen en cuerpos máficos o ultramáficos emplazados en diversos entornos geológicos. En edad, varían desde el Arcaico hasta el Terciario, pero el mayor número de depósitos son Arcaicos y Paleoproterozoico. Aunque estos yacimientos aparecen en la mayoría de los continentes, los yacimientos 
productivos (depósitos con tamaño y ley suficientes para ser económicos) son relativamente raros.

Los depósitos de sulfuros de níquel-cobre pueden aparecer como lentejones de sulfuros dentro de cuerpos máficos y/o ultramáficos. Típicamente, estos yacimientos poseen leyes de mineral entre $0.5 \%$ y $3 \%$ de níquel y entre $0.2 \%$ y $2 \%$ de cobre. Los tonelajes de depósitos individuales oscilan entre unas decenas de miles y decenas de millones de toneladas.

En los depósitos magmáticos de $\mathrm{Ni}$-Cu los sulfuros tienden a concentrarse en las partes inferiores de los cuerpos máficos o ultramáficos. La mineralización de sulfuros se puede dividir en diseminados y masivos, dependiendo de una combinación del contenido de sulfuros de la roca y la textura del silicato.

La mineralogía de los sulfuros de $\mathrm{Ni}-\mathrm{Cu}$ consta normalmente de un inter crecimiento de pirrotita, pentlandita y calcopirita. El cobalto, los PGE y el oro se extraen de la mayoría de los minerales magmáticos de $\mathrm{Ni}-\mathrm{Cu}$ como subproductos. Además, los depósitos pueden contener entre el $1 \%$ y $15 \%$ de magnetita asociada.

Relación modelo-tecnología de explotación: Aunque existen yacimientos subterráneos de $\mathrm{Ni}-\mathrm{Cu}$ magmáticos, los grandes depósitos por su magnitud y cercanía a la superficie se extraen a cielo abierto y en condiciones difíciles, como Norilsk y los canadienses. Casi siempre es el níquel el metal que provoca la explotación del conjunto (un precio hasta tres veces del cobre). Los metales acompañantes muchas veces son decisivos (cobalto y platinoides, sobre todo).

Las leyes de níquel y de cobre suelen ser semejantes. No es raro encontrar el concentrado global de $\mathrm{Ni}-\mathrm{Cu}$ por flotación (menor valor individual). El concentrado no suele pasar de un $15 \% \mathrm{Ni}-\mathrm{Cu}$. Ello es debido a los problemas de separación entre ambos metales y a la pérdida de recuperación.

\section{Los proyectos y sus indicadores}

En la situación actual, existen bastantes indicadores que nos hablan de la eficiencia técnica y económica de los proyectos en actividad o próximos a ella.Todos relacionan directa o indirectamente los resultados económicos con la eficacia técnica, y esta última se relaciona con la calidad natural del recurso mineral. Por ello, posee sentido tratar de contemplar las características usuales de un yacimiento (ley-tonelaje, geometría y profundidad, fundamentalmente) con su productividad económica. Por ello, la introducción de los modelos geológicos posee su sentido. Nos ayuda a clasificar los resultados en función de las variables naturales de los yacimientos.
Sin embargo, al hablar de los rendimientos económicos de un proyecto, necesitamos criterios para determinar de manera coherente y homogénea la variable precio del metal. Al analizar un proyecto minero, incluso antes de su diseño definitivo, es preciso fijar esta variable. De ahí se obtendrán, posteriormente, los indicadores necesarios. Pero, encontrar el precio adecuado significa fijar una cifra que reúna las condiciones de defensa ante la volatilidad de las cotizaciones con el suficiente conocimiento de la oportunidad en los mercados. Además de los factores de calidad del propio yacimiento, la experiencia y la adecuación de la tecnología disponible configurarán los criterios de elección de esta cifra.

Por tanto, es competencia de la empresa emprendedora decidir por la estrategia adecuada a su perfil de aceptación de riesgo y la formulación de los objetivos sobre la calidad del objeto a beneficiar, siempre bajo una mirada geológica experta (Espi, J.A. y de la Torre, L., 2013).

Para un precio determinado, le corresponde (aproximadamente) una ley límite o de corte concreta (riqueza en metal) que, realizada en ingresos obtenidos por el metal recuperable (NSR) compensa los costes de extracción y concentración mineral. Es decir, el "cut-off" nos proporciona la primera información acerca de la posible viabilidad del proyecto. Para cada cut-off se dispondrá de un nuevo yacimiento, ya que la ley límite marca la cantidad de toneladas que en los recursos geológicos disponibles superarán la riqueza fijada y, por lo tanto, serán susceptibles de aprovechamiento. Además, la acumulación de las toneladas que la superen fijará la ley media que, en realidad, constituye otro indicador de la calidad económica del yacimiento.

La relación entre la ley de corte o cut-off y el tonelaje producido nos define otra figura que gráficamente se denomina "relación ley-tonelaje" o sensibilidad del proyecto (Fig. 18). El análisis de esta relación ayuda a la comprensión de la fortaleza del proyecto ante variaciones importantes de la cotización del metal o circunstancias que hagan variar los costes de producción. Es más, para un precio del metal determinado, la diferencia entre las dos leyes mencionadas como indicadores (ley media y cut-off) produce una diferencia en metal que, en el fondo, traducido a unidades económicas, es el margen operativo del proyecto. Además, el EBITDA o capacidad potencial de generar fondos del proyecto, aproximadamente, resulta de multiplicar ese margen unitario operativo por el tonelaje producido en un año.

Las dos curvas que configuran la "relación leytonelaje," hoy se obtienen a partir del modelo de bloques dentro de un programa informático adecuado, $y$ 


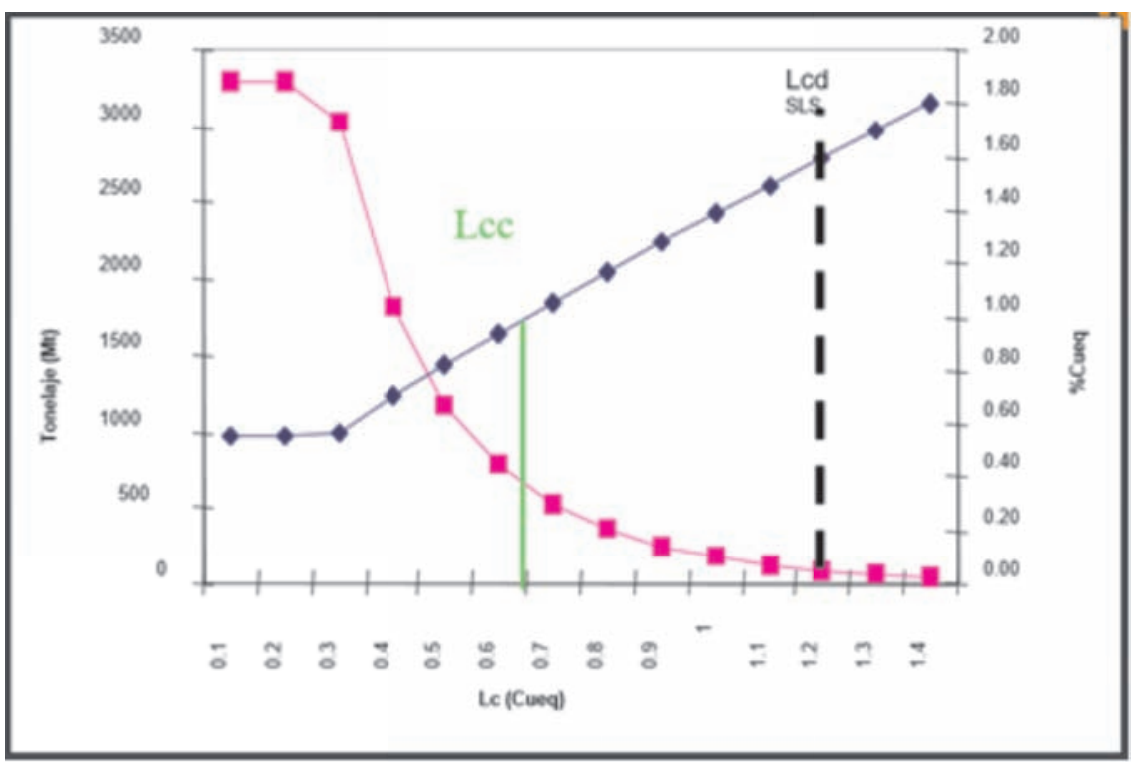

Figura 18. La curva ley-tonelaje de un proyecto minero se apoya en la ley de corte (Lc) que, a su vez dependerá de la cotización del metal para un periodo supuesto. De ella saldrán tanto la ley media Lcd y Lcc del yacimiento, como su correspondiente tonelaje. Todo ello se produce a partir del "modelo de bloques".

Figure 18. The grade-tonnage curve of a mining project is based on the cut-off grade (Lc) which, in turn, will depend on the metal price for a supposed period. From it will be obtained, both the average ore grades (Lcd and Lcc) and their corresponding tonnage. All this is obtained from the "block model.

su morfología procede de la propia naturaleza del yacimiento, es decir, muy relacionada con las tipologías o modelos metalogénicos considerados.

A fin de estudiar el posible agotamiento de las tipologías de minerales de cobre según el agotamiento o cambio de la situación económica de las tipologías de sus yacimientos más importantes, se analizaron un total de 120 explotaciones (15.2 Mtpa de cobre metal, es decir, el $80 \%$ producción global de cobre). Como indicadores de efectividad económica, en la Tabla 2 se han elegido los indicadores de la ley media de las reservas explotadas $(\% \mathrm{Cu})$ y el "cashcost" de cobre metal producido ( $\$ / \mathrm{lb} \mathrm{Cu}$, es decir, el coste de producción de una libra de cobre metal) en proyectos de gran envergadura correspondientes a tres horizontes temporales próximos: 2000-2010, 2011-2016 y proyectos de ejecución más allá del año 2017.

Los resultados no son del todo concluyentes: el descenso de ley o riqueza en cobre en el trascurso de estos 15 años, no queda reflejado en todas las tipologías de yacimientos. Probablemente los datos considerados se encuentran sesgados a causa de la introducción de proyectos con circunstancias particulares que han avanzado el descenso de la ley hace ya diez años. Sin embargo, estos resultados también nos hablan del esfuerzo realizado en los últimos tiempos en la reducción de los costes de producción, al mar- gen de las cotizaciones del cobre, que no han descendido de manera abultada. También hay que tener en cuenta la calidad de los yacimientos analizados que todavía no refleja de manera concluyente el descenso de la ley media de los nuevos proyectos.

\begin{tabular}{|l|l|l|l|l|l|}
\hline \multicolumn{2}{|c|}{ MEDIA } & VMS & \multicolumn{1}{|c|}{ SH } & POR & IOCG \\
\hline & Cash Cost $(\$ / \mathrm{lb})$ & 0.83 & 1.51 & 1.18 & 1.23 \\
\cline { 2 - 6 } & Ley $(\% \mathrm{Cu})$ & $3.30 \%$ & $1.90 \%$ & $0.90 \%$ & $1.60 \%$ \\
\hline \multirow{2}{*}{ uuturo>2017 } & Cash Cost $(\$ / \mathrm{lb})$ & & & 0.95 & \\
\cline { 2 - 6 } & Ley $\% \mathrm{Cu})$ & & & $0.67 \%$ & $1.20 \%$ \\
\hline \multirow{2}{2011-2016}{} & Cash Cost & 0.89 & 1.49 & 1.13 & 1.27 \\
\cline { 2 - 6 } & Ley $(\% \mathrm{Cu})$ & $3.71 \%$ & $1.90 \%$ & $1.01 \%$ & $1.66 \%$ \\
\hline \multirow{2}{2000-2010}{} & Cash Cost $(\$ / \mathrm{lb})$ & 1.03 & 2.85 & 1.36 & 1.07 \\
\cline { 2 - 6 } & Ley $(\% \mathrm{Cu})$ & $4.16 \%$ & $1.50 \%$ & $0.42 \%$ & $1.40 \%$ \\
\hline
\end{tabular}

Tabla 2. Resumen del resultado de analizar 120 proyectos recientes (a partir del 2010), expresando tanto su ley media (grade) como sus costes de producción de una unidad metálica (cash cost). Los depósitos minerales están clasificados en tres tipologías fundamentales: VMS (sulfuros masivos volcano-sedimentarios), SH (sediment hosted), POR (pórfidos de cobre) y IOCG (hidrotermales ricos en hierro).

Table 2. Results summary of analyzing 120 recent projects (from 2010), expressing both, their average grade and their metal unit production costs (cash cost). The mineral deposits are classified into three fundamental types: VMS (Sediment Hosted), POR (Porphyry Copper) and IOCG (Hydrothermal Iron rich). 


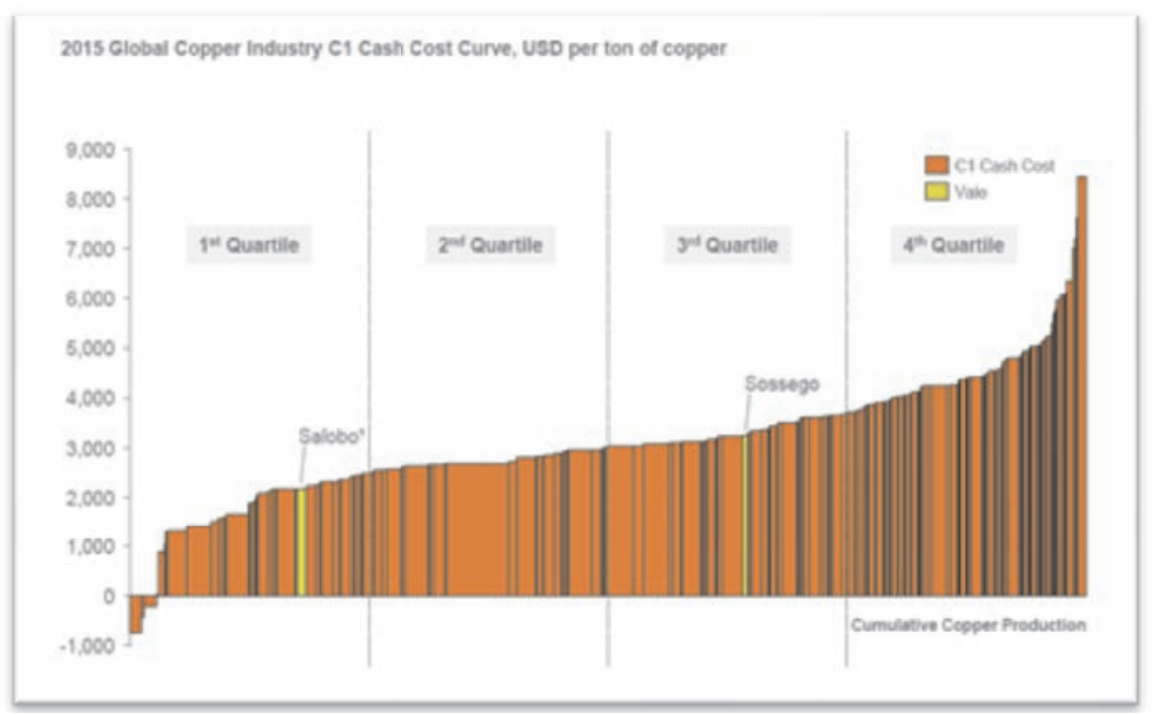

Figura 19. Posición de competitividad de dos proyectos mineros de cobre de la compañía Vale, 2015.

Figure 19. Competitive position of two Vale mining copper projects, 2015.

El "cash cost" de un proyecto es el mejor indicador de su efectividad económica respecto a su operatividad. Por ello, comparando este indicador con otros correspondientes a los actores del mercado, nos proporcionará una visión inigualable de su calidad operativa, tal como se muestra en el gráfico de la Figura 19. No obstante, hay que tener en cuenta que para cerrar la visión económica de un proyecto se ha de tener encuentro las inversiones necesarias para su actividad, es decir, se debe comparar el flujo económico marcado por la capacidad productiva y sus costes de operación, con los costes necesarios para que aquello ocurra, es decir, las inversiones iniciales y ahora también las de sostenimiento: AISC.

La mayoría de los nuevos proyectos mineros pretenden estar dentro del percentil 50 de la curva de costes (para el cobre, basado en \$/libra Cu metal o en $\$$ /tonelada de cobre producido), que es casi un requisito previo para la obtención de su financiación. Para llegar a esta posición, resulta necesario conseguir un proyecto con alta ley en cobre, o bien, un coste de operación muy bajo o una combinación de ambos.

Una ley elevada, o bien, los depósitos cercanos a la superficie, hoy resultan muy difíciles de conseguir, en especial los depósitos con una baja razón de desmonte en minería a cielo abierto, lo que permitiría disponer de unos bajos costos de operación.

Los depósitos más profundos requieren métodos de minería subterránea que, por lo general, significan mucho más altos costes de operación. En consecuencia, conseguir un proyecto dentro del percentil 50 de la curva de costes es cada vez más complicado, debido a que los yacimientos conocidos con leyes más altas son generalmente más profundos y los depósitos superficiales restantes todavía no desarrollados poseen una ley muy baja.

El resultado de todo esto es que, mientras que la demanda es incierta y las previsiones de crecimiento hacen que los precios a largo plazo se basen en el nivel de producción del percentil 90 (donde la curva de la oferta y la curva de demanda se cruzan). Existirán muy pocos proyectos que superen los obstáculos de, al menos, un 15\% TIR y, para la seguridad a largo plazo, que estén dentro del percentil 50 de la curva de costes.

Esto seguirá siendo la situación hasta que la demanda prevista recoja un período razonable, o bien, con el coste alto, los productores marginales desciendan en la curva de costes, lo cual no lo hace más fácil para los nuevos proyectos, a fin de conseguir el percentil 50 de la curva, cuestión verdaderamente complicada.

En cualquier caso, habrá un retraso considerable antes de que los proyectos que cumplan las condiciones anteriores estén listos para la producción, y este retraso resulta inevitable durante un tiempo, durante el cual, el suministro del metal se reducirá y la demanda aumentará gradualmente o incluso de manera drástica.

Esto conducirá a la siguiente subida del precio del metal. Lo único que es incierto en este escenario, tal como la historia ha registrado en varias ocasiones, es cuándo va a suceder.

Este mecanismo tiende a eliminar los proyectos con costes extremos superiores como consecuencia de las reducciones de precios cotizables y contempla 
la eliminación de los proyectos de costes bajos por agotamiento acelerado o bien por sus reducidas dimensiones.

Contemplamos como las leyes tienden a homogeneizarse en los ciclos económicos y los descensos en los valles de la evolución de los precios en los mercados de las materias primas minerales hacen el papel de selección y renovación de los grupos de calidad de anteriores ciclos.

De ahí pueden nacer tipologías de yacimientos desaparecidas o minoradas en su importancia en anteriores condiciones de entorno y que, con el precio de los metales al alza o a la baja, tratan de definir su papel en su nueva situación: selectividad en la demanda, condiciones de abastecimiento y criticidad de suministro, tecnologías emergentes, zonas nuevas antes prohibidas, etc.

\section{Factores Geológicos en el cobre y su futuro}

Según Stephen E. Kesler (2007), la población mundial está creciendo más rápidamente que en cualquier otro momento en la historia, y el consumo de minerales lo hace más rápidamente que la población, en tanto y cuanto nuevos consumidores entran en el mercado de minerales como consecuencia de su aumento en la calidad de vida. ¿Significa esto que nos enfrentaremos a una crisis de suministro de minerales en el siglo XXI? Si es así, podríamos resolver esta crisis de suministro de minerales con el aumento de la exploración de nuevos recursos minerales y, por ello, se requerirá una más fiable información geológica y un más fácil acceso al territorio. Las respuestas a estas preguntas deben basarse en las predicciones de la demanda mundial de minerales en el siglo $X X I$, junto con una mejor comprensión de la relación entre las reservas mundiales de minerales y la formación de los yacimientos.

En los últimos 50 años, y probablemente durante mucho más tiempo, las reservas minerales acumuladas han crecido más rápidamente que la producción. El comportamiento de los precios durante el siglo pasado confirma que las crecientes demandas han sido adecuadamente satisfechas. La producción histórica de minerales no ha agotado las reservas mundiales en la forma en que los depósitos individuales de mineral se agotan. Sin embargo, ha cambiado la forma y la naturaleza de la población mundial. Uno de los ejemplos que ejemplarizan estas afirmaciones se refiere a la producción del cobre.

Según J. Tilton y J.I. Guzmán (2017), la cuestión sobre si considerar los metales accesibles del Planeta como un stock totalmente fijo o la visión más amplia de un cúmulo metálico muy dependiente de la tecnología de acceso, es la cuestión fundamental que marca la presunción del acceso a los metales del futuro. Aceptando en este momento la existencia de un stock fijo, la escasez futura parece inevitable. Sin embargo, aceptando que el stock depende de la oportunidad de inversión en tecnología apropiada a los precios provocados por la presunción de escasez, los metales necesarios estarían accesibles en cada momento. Es decir, que los minerales y metales estarían disponibles, dependiendo de si los efectos de las reducciones de costes debidos a la aplicación de tecnologías innovadoras fueran mayores o menores que los efectos de los costes por la escasez de las materias primas minerales.

El mismo J.Tilton recalca que, en contra de los que a menudo se piensa, en el futuro el aumento inevitable de población y su correspondiente aumento en la demanda de los bienes naturales, no debe suponer la provocación de una escasez de los mismos, más bien lo contrario, ya que al mismo tiempo que se incrementa la demanda también existe un aumento del stock de capital humano, que debe ser capaz de mejorar las situaciones de posible escasez de materias primas.

Otra interesante consideración es la de que el aprovechamiento de los minerales se realiza, sobre todo en los depósitos de mayor calidad o de riqueza superior, teniendo en cuenta que la distribución de los mismos tiende a ser logarítmico normal, es decir, unos pocos participan en una proporción muy elevada en la producción total (J.L. Berry).

Además, hay que tener en cuenta que, en el sentido estadístico de la producción, existen dos modelos: el de los yacimientos derivados de singularidades geológicas (procesos de formación ligados a los fluidos movilizados por una fuente de energía capaz de atrapar y movilizar un stock metálico y depositarlo en condiciones de mayor concentración) y aquellos depósitos minerales derivados de los extremos de una distribución general en condiciones no tan exclusivas (Fig. 20)

A este último conjunto pertenecen los minerales denominados "bulk", de escaso valor unitario, pero de trascendencia económica extraordinaria: mineral de hierro, bauxitas, minerales de manganeso, cromitas, fosfatos, sales potásicas, etc. Al otro conjunto pertenecen el resto de minerales y metales valiosos, que se explotan en concentraciones mucho más inferiores. En esta visión, los minerales de volumen presentan posibilidades de extracción extraordinarias y, en muchos casos, la exigencia de alcanzar la riqueza necesaria para su explotación económica, no parece resentirse con el paso de los años. 


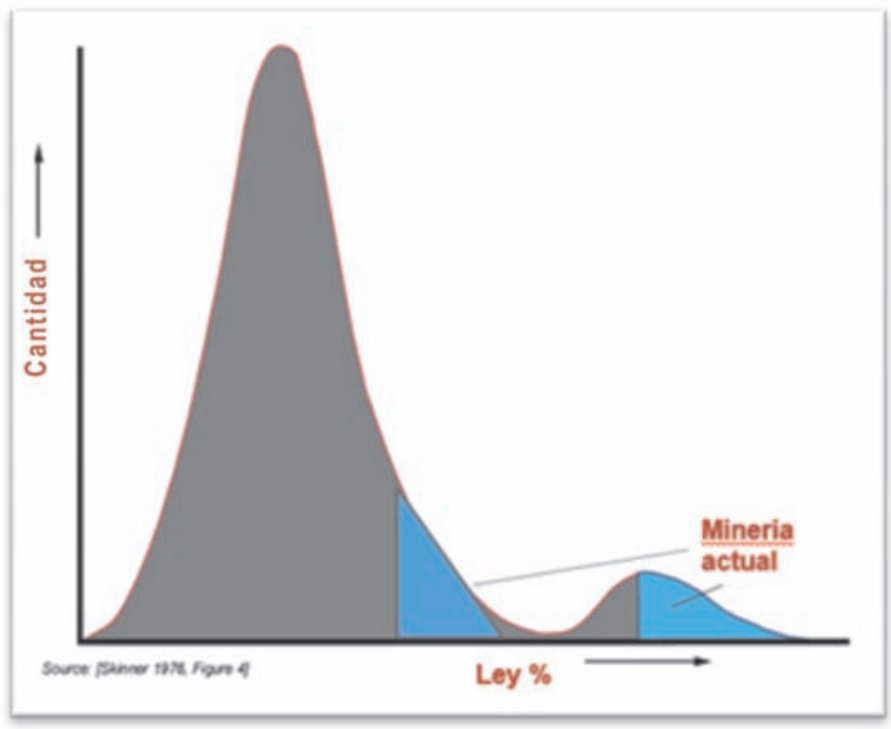

Figura 20. Distribución geoquímica de los elementos escasos en la corteza terrestre. Skinner 1976.

Figure 20. Geochemical distribution of scarce elements in the earth's crust. Skinner 1976.

El Servicio Geológico Americano, USGS, desde hace ya bastantes décadas analiza por modelos metalogenéticos su participación en la producción global $y$, sobre todo, en las características estadísticas de sus magnitudes fundamentales: la ley media y el tonelaje de los recursos. En prácticamente todas las tipologías se observa un ajuste más o menos satisfactorio al modelo logarítmico normal, es decir, que la holgura de la distribución presume oportunidades muy amplias al incremento de la producción en riquezas o concentraciones más modestas (ver Fig. 21).

\section{Buscando la sostenibilidad en el ámbito minero}

La minería del cobre, muchas veces acusada de gigantismo ante las explotaciones a cielo abierto de los pórfidos (recordemos explotaciones con gran movimiento de tierras, y una ley menguada hasta el $0.4 \% \mathrm{Cu}$ ), resulta a todas luces imprescindible para la sociedad en su progreso, pero, sin embargo, se debe mantener vigilante ante los desafíos de un siglo XXI que acusa unas prácticas medioambientales y sociales que deberán ser mejoradas en muchos casos. En una actividad tan controvertida como es actualmente la minería, las empresas mineras y los grupos de interés, entre los que se encuentra la sociedad como consumidora pero también como decisora, tienden a buscar la sostenibilidad en sus tres dimensiones, económica, social y medioambiental.

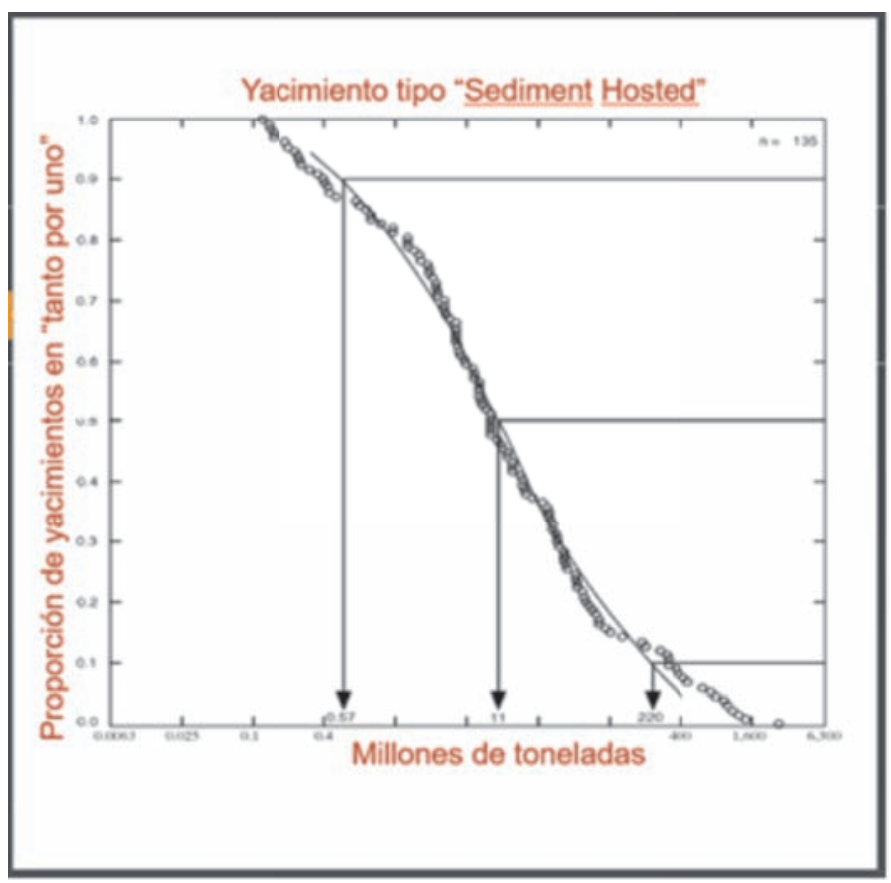

Figura 21. Curvas de frecuencia en tonelaje de los yacimientos mundiales del tipo "Sediment Hosted" del USGS. "n" es el número de depósitos considerados.

Figure 21. Tonnage frequency curves of the "Sediment Hosted" deposits from USGS global models. " $n$ " is the number of deposits considered.

Lograr una paridad económica con una apropiada distribución de riesgos y beneficios entre la compañía minera, el Gobierno y la percepción de las comunidades afectadas es necesario. El uso del suelo, cada vez sufre de mayor competencia, rivalizando con cultivos, parques nacionales o una urbanización creciente.

Los permisos de Evaluación de Impacto Ambiental (previos al inicio del proyecto), el control de Impacto Ambiental durante la operación (vertidos, residuos, gestión del agua valorando su uso y contaminación, los gases de efecto invernadero producidos en todo el ciclo), y la rehabilitación llegado el cierre de mina (aprobado en fase proyecto) convierten su efectividad en crítica para el impacto ambiental y social local. EI aumento de escala logra la reducción de costes económicos por unidad extraída, pero a la par necesita de mayor volumen por unas leyes decrecientes, mayores movimientos de tierra, consumos, vertederos, etc. (Mudd, G. M., 2009)

Únicamente mediante un uso sostenible o responsable de los recursos se logrará mantener nuestro mundo en la forma que lo conocemos, sin demonizar la minería, pero siempre observando sus límites, y permitiendo el diálogo progreso-tecnología-explotación de recursos-resiliencia medioambiental, y $\sin$ 


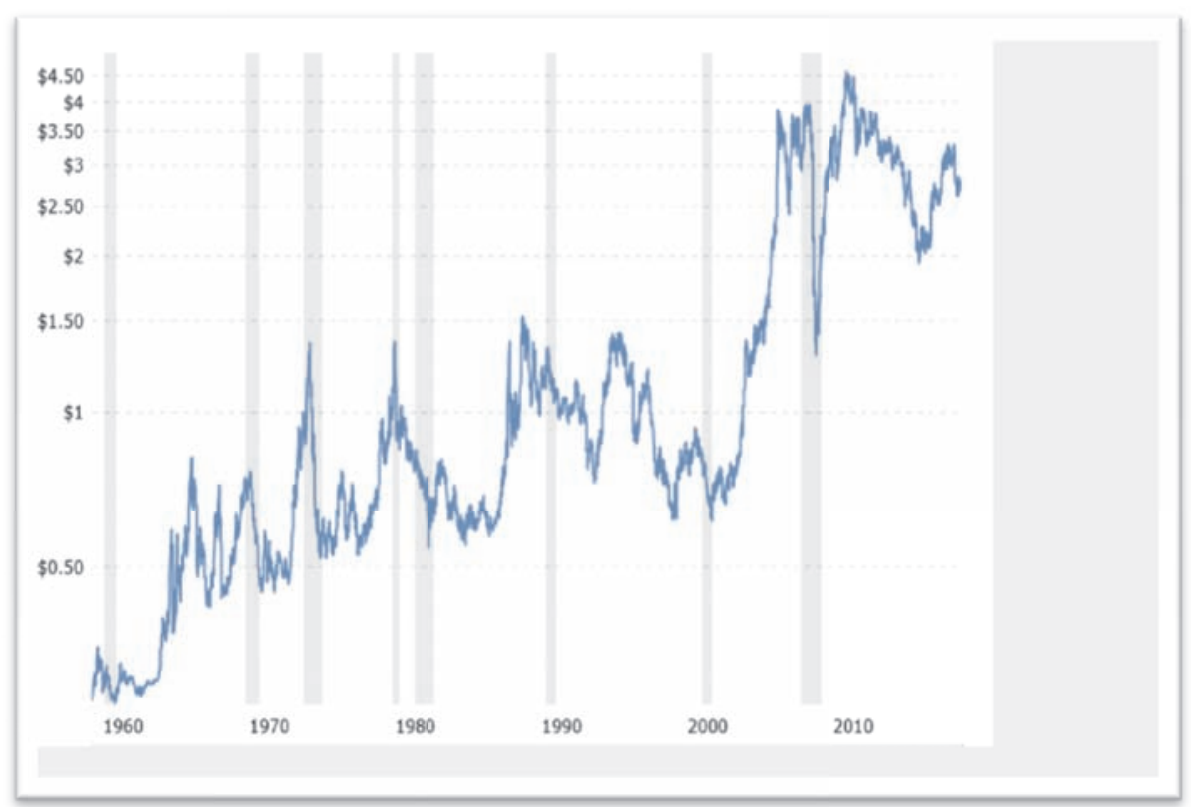

Figura 22. Evolución de los precios del cobre, en \$/lb Cu, en los ultimos 58 años. MACROTRENDS 2018.

Figure 22. Evolution of copper prices (\$/lb Cu) in the last 58 years. MACROTRENDS 2018.

paralizar su avance ante amenazas sobre el agotamiento de los recursos. (de la Torre, 2017).

\section{Discusión}

El analista internacional Mike Fulp describe en el año 2017 su visión de futuro basada en una gran experiencia y un buen conocimiento del mercado. Fulp comenta que el mercado se ha desequilibrado desde 2007 a causa de un pequeño déficit causado por las interrupciones del suministro (ver evolución de las cotizaciones en la Fig. 22).

La demanda de cobre ha crecido un $3.4 \%$ cada año desde que el mundo se electrificó en 1990. “Utilizábamos 4 millones de toneladas de cobre por año en 1960 y ahora estamos usando 23 millones de toneladas de cobre al año, y esto sigue creciendo ". Según Fulp, la demanda de cobre seguirá incrementándose. Eso se debe a que la población mundial continúa aumentando, al igual que el consumo de cobre por persona.

Según Fulp, la demanda de cobre aumentará en mercados emergentes como China, India, Brasil e Indonesia, así como en Estados Unidos. A medida que crezca la demanda, el déficit de oferta seguirá incrementándose. "No hay una solución fácil para el problema de suministro de cobre ". Según el analista, es difícil pensar de dónde vendrá el metal rojo. África tiene grandes fuentes de cobre, pero también tiene muchos países políticamente inestables. La clave, en palabras de Fulp, es ser más efectivo en la exploración, ya que "la tecnología y la innovación ayudarán a resolver el problema del suministro de cobre". [...]

\section{Conclusiones}

La evolución futura de los suministros se torna cada vez más compleja y los modelos de evolución son más escasos y de fiabilidad muy limitada.

Existe una enorme convergencia a nivel experto sobre la necesidad de un conocimiento geológico global suficiente para proceder a la elaboración de modelos sobre oferta y demanda de todos los metales, y más aún, en el caso del cobre.

Resulta mucho más abundante la opinión de que los recursos minerales situados en una posición hoy accesible poseen tal abundancia que, a pesar de las situaciones temporales de cierta escasez, no llegan a una calificación crítica. Esta situación sí que existe en algunos casos debido a otros elementos que entran dentro del sistema productivo. En modo alguno por agotamiento del stock mineral.

\section{Referencias}

Ayres R. U. 2001. Resources, Scarcity, Growth and the Environment. Center for the Management of Environmental Resources. INSEAD. April 2001. 
Banco Mundial. 2017. Informe Trimestral, abril 2017, hhtp://www.worldbank.org/commodities;pubdocs.world bank.org/en/ 174381493046968144/CMO-April-2017-FullReport.pdf)

Cox D. P. and Singer D.A. editors.1992. Mineral Deposit Models. US. Geological Survey Bulletin 1693.

Cox D. P., Lindsey D.A. Singer D.A. and Diggles M.F. Sediment-Hosted Copper Deposits of the World: Deposit Models and Database. U.S. Geological Survey Open-File Report 03-107.

Crowson P. 2011. The Copper Conundrum. Mining Magazine. 14-21. November 2011

Cuddington J. y Jerret D. 2008. Supercycles in Real Market Prices? IMF Staff Papers, Vol 55, N4, 541-565.

De la Torre, L., 2017. El progreso y la paradoja de los recursos naturales. La amenaza del agotamiento, Sistema $\mathrm{n}^{\circ} 247$

Dennis P. Cox, David A. Lindsey, Donald A. Singer, Barry C. Moring, and Michael F. European, Copper Institute, 2015. Annual Report.

Diggles. 2007. Sediment-Hosted Copper Deposits of the World: Deposit Models and Database. USGS. Open-File Report 03-107 Version 1.3 2003, revised 2007 Available online at http://pubs.usgs.gov/of/2003/of03-107/

Durán P y Tapia J. 2015. Depósitos de Sulfuros Masivos Vulcanogénicos (VMS) https://docplayer.es/41993269Depositos-de-sulfuros-masivos-volcanogenicos-vmssemestre-primavera-2015-paulina-duran-joselinetapia.html

Fischer-Kowalsky, M. y Haberl, H. 2000. El metabolismo socioeconómico, Ecología Polí-tica, nº 19.

Fischer-Kowalsky, M. y Swilling M. 2011. Decoupling natural resource use and environmen $\neg$ tal impacts from economic growth, Report of the Working Group on Decoupling to the International Resource Panel (UNEP).

Fletcher R. y Rammelt C. 2017. Decoupling: A Key Fantasy of the Post-2015 Sustainable Development Agenda, Globalizations, 14:3, 450-467.

Fulp M. 2017. “Mickey Fulp's Call on Copper". Investing News Network. May 30.

Gordon, R.B., M. Bertram and T.E. Graedel. 2006. Metal stocks and sustainability, Proceedings of the National Academy of Sciences, Vol. 103, No. 5, pp. 1209-1214.

Harmsen, J., Roes, A., Patel M. 2013. The impact of copper scarcity on the efficiency of 2050 global renewable energy scenarios, Energy, Elsevier, Vol. 50.

Jahanshahi, S., 2006. Assessing the environmental Impact of metal production processes, Journal of Cleaner Production, October, UNSW.

John D.A., editor. 2010. Porphyry Copper Deposit Model. Chapter B of Mineral Deposit Models for Resource Assessment2010. Porphyry Copper Deposit Model. Scientific Investigations Report 2010-5070-B

Kesler S.E. 2007. Mineral Supply and Demand into the 21st Century. Deposit Modeling, Mineral Resources Assessment and their Role in Sustainable Development. p.55-62. USGS Circular 1294

Kraft, J. 1978. On the Relationship Between Energy and GNP, Journal of Energy and Development, 3, pp. 401403.
Krauze E. 2016. Personas e Ideas, Penguin Random House, Méjico D.F.

Lambert I.B. (2001) Mining and sustainable development: considerations for minerals supply. Group Manager, National Projects and Advice. Australian Geological Survey Organization. Natural Resources Forum, JNRF

Lin, B. y Liu, C. 2016. Why is electricity consumption inconsistent with economic growth in China?, Energy Policy Volume 88, January 2016, pp. 310-316

Lydon J.W. 1984. Ore deposit models. Volcanogenic massive sulphide deposits. Geoscience Canada 11(4) p. 95-202

McGowan R.R., Roberts S., Foster R.P., Boyce A.J., Coller D. 2003. Origin of the copper-cobalt deposits of the Zambian Copperbelt: An epigenetic view from Nchanga, Geology 31 (6), p. 497-500.

McKinsey Global Institute. 2017. Beyond the supercycle: How technology is reshaping resources

ining Journal. 2010. Exploration Special. 2010. The search for metals and minerals

Mosier. D.L., Berger V.I., and Singer D.A. 2009. Volcanogenic Massive Sulfide Deposits of the World- Open-File Report 2009-1034. Database and Grade and Tonnage Models. U.S. Geological Survey.

Moreno S.A. 2006. Valoración de las cadenas productivas de la minería metálica global, usando herramientas innovadoras de gestión ambiental. Tesis Doctoral: Departamento de Ingeniería Geológica de la UPM.

Moreno S.A. y Espi J.A. 2007. Introducción al uso de las Herramientas de Gestión Ambiental aplicadas a los Recursos Naturales no renovables. Master Int. Aprovechamiento Sostenible de los Recursos Minerales. Red Desir. Programa Alfa. OEI

Mudd, G. M. 2009. The Sustainability of Mining in Australia: Key Production Trends and Their Environmental Implications for the Future. Research Report No RR5, Department of Civil Engineering, Monash University and Mineral Policy Institute, Revised - April 2009.

Nooten G.A. 2007. Sustainable Development and Nonrenewable Resources-A Multilateral perspective. Mineral Resources Assessment and Their Role in Sustainable Development. p.35-40. USGS Circular 1294

Northey S., Mohrb S., Mudda G.M., Wenga Z. and Giurcobet D. 2014. Modelling future copper ore grade decline based on a detailed assessment of copper resources and mining. Resources, Conservation and Recycling 83 Elsevier (2014) pp.190-201

PwC. 2016. Mine 2016. Slower, lower... but not defeated. www.pwc.com

PwC, 2017. Mine 2017. Stop. Think... Act. www.pwc.com

Rufael Y. 2014. Electricity consumption and economic growth in transition countries: A revisit using bootstrap panel Granger causality analysis Author links open overlay panel, Energy Economics, Volume 44, July 2014, pp. 325-330.

Schodde R. 2010. MEMS Conference Mineral and Metal Markets over the Long Term, Joint program with the SME annual meeting in Phoenix

Sheng-Tung, C. et al. 2007. The relationship between GDP 
and electricity consumption in 10 Asian countries, Energy Policy, Volume 35, Issue 4, pp. 2611-2621.

Singer D.A. 1975. Geology and resources of copper deposits. Washington. US Geological Survey

Skinner B.J. 1976. A second iron age ahead? American Scientist 64 May-June 1976. pp 258-269.

Tilton, J. 2001. Mining, Minerals and Sustainable Development, IIED.

Tilton J.E. and Guzmán J.I., 2017. Mineral Economics and Policy. Editorial Advisers Press. 255p.

Tilton J.E. 2001. MMSD. N 14 Depletion and the Long-run Availability of Mineral Commodities. Colorado School of Mines. IIED. March 2001

Tilton, J.E. 2001. Depletion and the Long-run Availability of Mineral Commodities. Mining, Minerals and Sustainable Development, 14.

Tilton, J.E., and G. Lagos. 2007. Assessing the long-run availability of copper, Resources Policy, Vol. 32, pp. 19-23.

USGS, 2008. Porphyry Copper deposit models USGS.
USGS. 2014. USGS regional copper resource assessments, http://minerals.usgs.gov/global

U.S. Geological Survey. 2017. Mineral Commodity Summaries. Preliminary Model of Porphyry Copper Deposits, January 2017. U.S. Geological Survey. OpenFile Report 2008-1321

Vázquez F. 2012. Manual de yacimientos minerales. U.D. Proyectos. ETSI Minas-UPM. Madrid

Weizsäcker E. 2014. Decoupling 2: technologies, opportunities and policy options, Nairobi, (UNEP).

Wellmer FW. and Becker-Platen J. D. 2002. Sustainable development and the exploitation of mineral and energy resources: a review. Int. J Earth Sci (Geol Rundsch) (2002) 91:723.

Wellmer F.W., Dieter J.B. 2007. Global Nonfuel Mineral Resources and Sustainability. Proceedings for a Workshop on Deposit Modeling, Mineral Resources Assessment and Their Role in Sustainable Development. p.1-16. USGS Circular 1294

Recibido: diciembre 2017

Revisado: febrero 2018

Aceptado: junio 2018

Publicado: marzo 2019 
